\title{
Review
}

\section{A Survey of Several Finite Difference Methods for Systems of Nonlinear Hyperbolic Conservation Laws}

\author{
GARY A. SOD \\ Courant Institute of Mathematical Sciences, New York University, New York, New York 10012, \\ and Lawrence Livermore Laboratory, P. O. Box 808, Livermore, California
}

\begin{abstract}
The finite difference methods of Godunov, Hyman, Lax and Wendroff (two-step), MacCormack, Rusanov, the upwind scheme, the hybrid scheme of Harten and Zwas, the antidiffusion method of Boris and Book, the artificial compression method of Harten, and Glimm's method, a random choice method, are discussed. The methods are used to integrate the one-dimensional Eulerian form of the equations of gas dynamics in Cartesian coordinates for an inviscid, nonheat-conducting fluid. The test problem was a typical shock tube problem. The results are compared and demonstrate that Glimm's method has several advantages.
\end{abstract}

\section{INTRODUCTION}

In the past few years numerous methods have been developed for solving systems of nonlinear hyperbolic conservation laws

$$
\mathbf{U}_{t}+\mathbf{F}(\mathbf{U})_{x}=0,
$$

where $\mathbf{F}$ is a smooth function on $R^{n}$ of $\mathbf{U}$ and the derivative matrix $D F(U)$ possesses strictly nonlinear or linearly degenerate eigenfunctions and eigenvalues in the sense of Lax [16]. In the terminology of gas dynamic this corresponds to systems having modes which admit respectively, discontinuities of shock type, or contact discontinuities, only. For example, a single equation

$$
u_{t}+g(u)_{x}=0,
$$

is strictly nonlinear if $g(u)_{x x} \neq 0$ and is linearly degenerate if $g(u)=C u$, where $C$ is a constant.

There are several difficulties in solving such systems numerically. Inherent in any finite difference scheme is an assumption on the regularity of the solution. Typically such schemes produce oscillations behind a shock. All finite difference schemes have numerical diffusion, dispersion, or both due to the truncation error. As a result there will be diffusion across the contact discontinuity with each time step, causing the contact discontinuity to be smeared in the course of the calculation. 
A shock is also smeared, though less dramatically. Finally, there is often difficulty in obtaining an accurate approximation to the smooth parts of the solution, particularly at points where certain higher order derivatives fail to exist.

The finite difference methods of Godunov [6], Hyman [13], Lax and Wendroff [19], MacCormack [18], Rusanov [21], the upwind scheme [20], and the hybrid scheme of Harten and Zwas [12] will be discussed. Glimm's method, a random choice method, will also be considered. Glimm's method at first appearance may not seem to fit in the category of the other methods. However, it shares several features with the above difference schemes. All of these methods are fixed grid methods having the same grid structure. These methods require about the same amount of storage. All of the above finite difference schemes are finite difference approximations to the derivative arising in the conservation laws and can treat in principle an arbitrary system of conservation laws. Similarly Glimm's method can treat an arbitrary system of conservation laws. See Harten and Sod [11].

The finite difference schemes as well as Glimm's method share one other feature, namely, a family of waves interacting can be handled automatically. However, the degree of resolution to which such interactions are represented is not the same for all methods.

Most finite difference methods when applied to problems with discontinuities produce oscillations behind the shock. Von Neumann and Richtmyer [26] developed an artificial viscosity term which was introduced into the Lagrangian form of the equations of gas dynamics. The goal of the artificial viscosity was to reduce the oscillations while allowing the shock transition to occupy only a few mesh points and having negligible effect in the smooth regions. All other forms of artificial viscosity are variations of the one introduced by von Neumann and Richtmyer. The one used in this survey due to Lapidus [14] is no exception. However, it is suited to the conservation laws and can be added to an existing method as a fractional step. One important feature of Lapidus' form of artificial viscosity is that a variant of it can be applied to general conservation laws. Artificial viscosity will not spread a contact discontinuity (see section on artificial viscosity or Sod [25]). The smearing of a contact discontinuity is due to the truncation error of the scheme and the smearing of a shock is due to the truncation error of the scheme as well as the artificial viscosity. The spreading of the contact discontinuity being much more severe, $O\left(n^{1 /(q+1)}\right)$ for a $q$ th order accurate scheme, where $n$ is the number of time steps. See Harten [8].

Recently methods have been developed for correcting the smearing of shocks and contact discontinuities. The "flux corrected transport" method of Boris and Book [1] is used to obtain a high degree of resolution without oscillations. The artificial compression method of Harten [8-10] is designed to sharpen results in regions containing shocks and contact discontinuities.

The inviscid, non-heat-conducting equations of gas dynamics can be written in conservation form with the flux $\mathbf{F}$ having the form discussed above. However, there is a large class of problems for which little is known. This is the case where $\mathbf{F}$ is either nonsmooth or not strictly nonlinear. Examples are the Buckley-Leverett equation, in 
the case of a single equation (see Buckley and Leverett [27]) and the problem of an exothermic reacting gas, in the case of systems. In these cases special methods have to be considered. For the case of an exothermic reacting gas see Chorin [4] and Sod [24].

The only "high"-order method considered in this survey is the hybrid scheme of Harten and Zwas. Fourth- and sixth-order methods were originally considered for the equations of gas dynamics. However, with all of the high-order methods using the appropriate high-order artificial viscosity term, the coefficient of numerical diffusion was so large that the time stcp became prohibitivcly small. The only way in which a solution could be obtained with these high-order methods was to use the artificial viscosity due to Lapidus, which is third order. With the use of a low-order artificial viscosity term, all benefits obtained by using a high-order method are lost.

In the following sections a brief discussion of the methods is given, their solution to a sample one-dimensional problem is compared, and the merits of the methods are discussed. Due to the nonstandardness of Glimm's method, as well as the difficulty in programming, its acceptance as an effective and efficient numerical tool may be restricted. For this reason the equations used by Glimm's method are derived and a flow chart for its implementation is given. For further details on these methods see Sod [25].

\section{Basic Equations}

The one-dimensional equations of gas dynamics may be written in the (conservation) form:

$$
\begin{aligned}
\partial_{t} \rho+\partial_{x}(\rho u) & =0, \\
\partial_{t} m+\partial_{x}\left(\left(m^{2} / \rho\right)+p\right) & =0, \\
\partial_{t} e+\partial_{x}((m / \rho)(e+p)) & =0,
\end{aligned}
$$

where $\rho$ is the density, $u$ is the velocity, $m=\rho u$ is momentum, $p$ is pressure, and $e$ is energy per unit volume. We may write $e=\rho \epsilon \mid \frac{1}{2} \rho u^{2}$, where $\epsilon$ is the internal energy per unit mass. Assume the gas is polytropic, in which case

$$
\epsilon=p /(\gamma-1) \rho,
$$

where $\gamma$ is a constant greater than one. Furthermore, from (3) we have

$$
p=A(S) p^{\nu},
$$

where $S$ denotes entropy.

Equations (1)-(3) may be written in vector form

$$
\mathbf{U}_{t}+\mathbf{F}(\mathbf{I})_{x}=\mathbf{0},
$$

where

$$
\mathbf{U}=\left[\begin{array}{c}
\rho \\
m \\
e
\end{array}\right] \quad \text { and } \quad \mathbf{F}(\mathbf{U})=\left[\begin{array}{c}
m \\
\left(m^{2} / \rho\right)+p \\
(m / \rho)(e+p)
\end{array}\right]
$$


In order to deal with solutions containing shocks we write the equations in integral form, which is obtained by integrating Eqs. (2a)-(2c) (or Eq. (5)) over any region in the upper half of the $(x, t)$ plane and applying Green's theorem

$$
\begin{aligned}
\int \rho d x+\int m d t & =0, \\
\int m d x+\int\left(\left(m^{2} / \rho\right)+p\right) d t & =0, \\
\int e d x+\int((m / \rho)(e+p)) d t & =0 .
\end{aligned}
$$

\begin{tabular}{|c|c|c|c|}
\hline Originator & Order & Scheme & Stability ${ }^{a}$ \\
\hline Godunov & 1 & $\begin{array}{l}\overline{\mathbf{u}}_{i+\frac{1}{2}}^{n+1}=\frac{1}{2}\left(\mathbf{u}_{i+1}^{n}+\mathbf{u}_{i}^{n}\right)-(\Delta t / \Delta x)\left(\mathbf{F}_{i+1}^{n}-\mathbf{F}_{i}^{n}\right) \\
\mathbf{u}_{i}^{n+1}=\mathbf{u}_{i}^{n}-(\Delta t / \Delta x)\left(\overline{\mathbf{F}}_{i+\frac{1}{2}}^{n+1}-\overline{\mathbf{F}}_{i-\frac{1}{2}}^{n+1}\right)\end{array}$ & $o \leqslant 1$ \\
\hline $\begin{array}{l}\text { Lax-Wendroff } \\
\text { (two-step) }\end{array}$ & 2 & $\begin{array}{l}\mathbf{u}_{i+\frac{1}{2}}^{n+\frac{1}{2}}=\frac{1}{2}\left(\mathbf{u}_{i+1}^{n}+\mathbf{u}_{i}^{n}\right)-(\Delta t / 2 \Delta x)\left(\mathbf{F}_{i+1}^{n}-\mathbf{F}_{i}^{n}\right) \\
\mathbf{u}_{i}^{n+1}=\mathbf{u}_{i}^{n}-(\Delta t / \Delta x)\left(\mathbf{F}_{i+\frac{1}{2}}^{n+\frac{1}{2}}-\mathbf{F}_{i-\frac{1}{2}}^{n+\frac{1}{2}}\right)\end{array}$ & $\sigma \leqslant 1$ \\
\hline MacCormack & 2 & $\begin{array}{l}\overline{\mathbf{u}_{i}^{n+1}}=\mathbf{u}_{i}^{n}-\left(\Delta t_{i} \Delta x\right)\left(\mathbf{F}_{i+1}^{n}-\mathbf{F}_{i}{ }^{n}\right) \\
\mathbf{u}_{i}^{n+1}=\frac{1}{2}\left(\mathbf{u}_{i}^{n}+\overline{\mathbf{u}_{i}^{n+1}}\right)-(\Delta t / 2 \Delta x)\left(\overline{\mathbf{F}_{i}^{n+1}}-\overline{\mathbf{F}_{i-\mathbf{1}}^{n+1}}\right)\end{array}$ & $\sigma \leqslant 1$ \\
\hline Rusanov & 1 & $\begin{aligned} & \mathbf{u}_{i}^{n+1}= \mathbf{u}_{i}{ }^{n}-(\Delta t / 2 \Delta x)\left(\mathbf{F}_{i+1}^{n}-\mathbf{F}_{i-\mathbf{1}}^{n}\right) \\
&+\frac{1}{4}\left(\left(\alpha_{i+1}^{n}+\alpha_{i}^{n}\right)\left(\mathbf{u}_{i+1}^{n}-\mathbf{u}_{i}^{n}\right)\right. \\
&\left.-\left(\alpha_{i}^{n}-\alpha_{i-1}^{n}\right)\left(\mathbf{u}_{i}^{n}-\mathbf{u}_{i-1}^{n}\right)\right) \\
& \alpha_{i}^{n}=\omega(\Delta t / \Delta x)(u+c)_{i}{ }^{n}\end{aligned}$ & $\begin{array}{l}\sigma \leqslant 1 \\
\sigma \leqslant \omega \leqslant 1 / \sigma\end{array}$ \\
\hline Upwind & 1 & 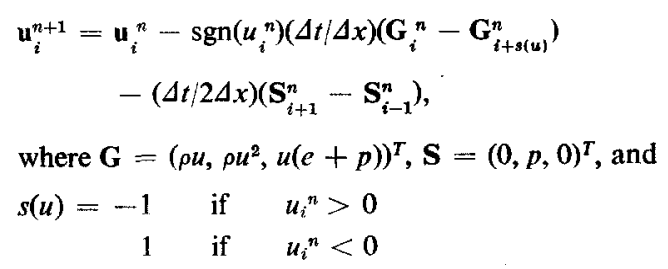 & $\sigma \leqslant 1$ \\
\hline
\end{tabular}

TABLF I

Standard Finite Difference Methods

$a_{\sigma}=\max (|u|+c) \Delta t / \Delta x$, where $c$ denotes the local sound speed. 


\section{DesCRIPTION OF THE METHODS}

The methods of Godunov [6], Lax and Wendroff (two-step) [19], MacCormack [18], Rusanov [21], and the upwind difference scheme [20] have been widely used and no benefit can be obtained by describing them here. Hence, these schemes will merely be listed in Table I. The remaining methods under consideration will be briefly discussed.

Consider the nonlinear system of Eqs. (5). Divide time into intervals of length $\Delta t$ and let $\Delta x$ be the spatial increment. The solution is to be evaluated at time $n \Delta x$, where $n$ is a nonnegative integer at the spatial increments $i \Delta x, i=0, \pm 1, \pm 2, \ldots$, and at time $\left(n+\frac{1}{2}\right) \Delta t$ at $\left(i+\frac{1}{2}\right) \Delta x$. Let $\mathbf{u}_{i}{ }^{n}$ approximate $\mathbf{U}(i \Delta x, n \Delta t)$ and $\mathbf{u}_{i+\frac{1}{2}}^{n+\frac{1}{2}}$ approximate $\mathbf{U}\left(\left(i+\frac{1}{2}\right) \Delta x,\left(n+\frac{1}{2}\right) \Delta t\right)$.

\section{Glimm's Method}

Recently the random choice method introduced by Glimm [7] has been developed for hydrodynamics by Chorin [3]. The method is a two-step method. To find $\mathbf{u}_{i+t}^{n+t}$ and thus define the method, consider system (5) along with the piecewise constant initial data

$$
\begin{aligned}
\mathbf{U}(x, n \Delta t) & =\mathbf{u}_{i+1}^{n}, & & x \geqslant\left(i+\frac{1}{2}\right) \Delta x, \\
& =\mathbf{u}_{i}{ }^{n}, & & x<\left(i+\frac{1}{2}\right) \Delta x .
\end{aligned}
$$

This defines a sequence of Riemann problems. If $\Delta t<\Delta x / 2(|u|+c)$, where $c$ is the local sound speed, the waves generated by the different Riemann problems will not interact. Hence the solution $\mathbf{v}(x, t)$ to the Riemann problem can be combined into a single exact solution. Let $\xi_{n}$ be an equidistributed random variable which is given by the Lebesgue measure on the interval $\left[-\frac{1}{2}, \frac{1}{2}\right]$. Define

$$
\mathbf{u}_{i+\frac{1}{2}}^{n+\frac{1}{2}}=\mathbf{v}\left(\left(i+\xi_{n}\right) \Delta x,\left(n+\frac{1}{2}\right) \Delta t\right) .
$$

At each time step, the solution is approximated by a piecewise constant function. The solution is then advanced in time exactly and the new values are sampled. The method depends on the possibility of solving the Riemann problem exactly and inexpensively. Chorin [3] (see also Sod [22]) modified an iterative method due to Godunov [5] which will be described below.

Consider system (5) with the initial data

$$
\begin{aligned}
\mathbf{U}(x, 0) & =S_{l}=\left(\rho_{l}, u_{l}, p_{l}\right), & & x<0, \\
& =S_{r}=\left(\rho_{r}, u_{r}, p_{r}\right), & & x \geqslant 0 .
\end{aligned}
$$

The solution at later times looks like (see [15]) Fig. 1, where $S_{1}$ and $S_{2}$ are either a shock or a centered rarefaction wave. The region $S_{*}$ is a steady state. The lines $l_{1}$ and $l_{2}$ separate the states. The contact discontinuity $d x / d t=u_{*}$ separates the state $S_{*}$ into two parts with possibly different values of $\rho_{*}$, but equal values of $u_{*}$ and $p_{*}$. 


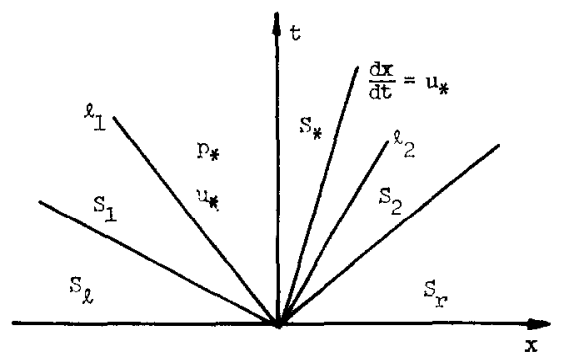

Fig. 1. Solution of a Riemann problem.

Using this iterative method we first evaluate $p_{*}$ in the state $S_{*}$. Define the quantity

$$
M_{l}=\left(p_{l}-p_{*}\right) /\left(u_{l}-u_{*}\right) .
$$

If the left wave is a shock, using the jump condition $U_{l}[\rho]=[\rho u]$, we obtain

$$
M_{l}=\rho_{l}\left(u_{l}-U_{l}\right)=\rho_{*}\left(u_{*}-U_{l}\right),
$$

where $U_{l}$ is the velocity of the left shock and $\rho_{*}$ is the density in the portion of $S_{*}$ adjoining the left shock. Similarly, define the quantity

$$
M_{r}=\left(p_{r}-p_{*}\right) /\left(u_{r}-u_{*}\right) .
$$

If the right wave is a shock, using the jump conditions $U_{r}[\rho]=[\rho u]$, we obtain

$$
M_{r}=-\rho_{r}\left(u_{r}-U_{r}\right)=-\rho_{*}\left(u_{*}-U_{r}\right),
$$

where $U_{r}$ is the velocity of the right shock and $\rho_{*}$ is the density in the portion of $S_{*}$ adjoining the right shock.

In either of the two cases ((12) or (13) for $M_{l}$ and (14) and (15) for $M_{r}$ ) we obtain

$$
\begin{aligned}
M_{r} & =\left(\rho_{r} p_{r}\right)^{1 / 2} \phi\left(p_{*} / p_{r}\right), \\
M_{l} & =\left(\rho_{l} p_{l}\right)^{1 / 2} \phi\left(p_{*} / p_{l}\right),
\end{aligned}
$$

where

$$
\begin{aligned}
\phi(x) & =\left(\frac{\gamma+1}{2} x+\frac{\gamma-1}{2}\right)^{1 / 2}, & & x \geqslant 1, \\
& =\frac{\gamma-1}{2 \gamma^{1 / 2}} \frac{1-x}{1-x^{\frac{1}{2-\frac{1}{2} \gamma}}}, & & x \leqslant 1 .
\end{aligned}
$$

Upon elimination of $u_{*}$ from (12) and (14) we obtain

$$
p_{*}=\left(u_{l}-u_{r}+\frac{p_{r}}{M_{r}}+\frac{p_{l}}{M_{l}}\right) /\left(\frac{1}{M_{l}}+\frac{1}{M_{r}}\right) .
$$


Equations (16a), (16b), and (18) represent three equations in three unknowns for which it can be seen that there exists a real solution. Upon choosing a starting value $p_{*}{ }^{0}$ (or $M_{l}^{0}$ and $M_{r}{ }^{0}$ ), we iterate using Eqs. (16a), (16b), and (18). For details of the starting values see Chorin [3] and Sod [25].

After $p_{*}, M_{l}$, and $M_{r}$ have been determined we may obtain $u_{*}$ by eliminating $p_{*}$ from Eqs. (12) and (14),

$$
u_{*}=\left(p_{l}-p_{r}+M_{l} u_{l}+M_{r} u_{r}\right) /\left(M_{l}+M_{r}\right)
$$

from which the complete solution of the Riemann problem can be determined using the jump conditions for shock waves and the isentropic law and constancy of the Riemann invariants for the rarefaction waves. See the Appendix.

The finite difference method due to Godunov [6] in Table $I$ is for the Eulerian form of the equations of gas dynamics. The method developed by Godunov [5] for the Lagrangian form is also a two-step method where the second step is the second half step in Table I. However, the values of $u_{i+\frac{1}{2}}^{n+\frac{k}{2}}$ and $p_{i+\frac{1}{2}}^{n+\frac{1}{2}}$ are replaced by $u_{*}$ (19) and $p_{*}$ (18) from the Riemann problem at $i+\frac{1}{2}$.

\section{Artificial Viscosity}

In the methods of Godunov, MacCormack, and Lax and Wendroff (two-step), an artificial viscosity term was added. The artificial viscosity term used was introduced by Lapidus [14]. It has the advantage that it is very easy to add to an existing scheme and it retains the high-order accuracy of the scheme. Let $\tilde{\mathbf{u}}_{i}^{n+1}$ be the approximation at time $(n+1) \Delta t$ obtained by any one of the above schemes. This value is replaced by the new approximation

$$
\mathbf{u}_{i}^{n+1}=\tilde{\mathbf{u}}_{i}^{n+1}+\frac{\nu \Delta t}{\Delta x} \Delta^{\prime}\left[\left|\Delta^{\prime} \tilde{\mathbf{u}}_{i+1}^{n+1}\right| \cdot \Delta^{\prime} \tilde{\mathbf{u}}_{i+1}^{n+1}\right]
$$

where $\Delta^{\prime} \tilde{\mathbf{u}}_{i}{ }^{n}=\tilde{\mathbf{u}}_{i}{ }^{n}-\tilde{\mathbf{u}}_{i-1}^{n}$ and $\nu$ is an adjustable constant.

Equation (20) is a fractional step for the numerical solution of the following diffusion equation

$$
\mathbf{U}_{t}=(\nu \Delta t / \Delta x)\left(\Delta x^{3}\right)\left[\left|u_{x}\right| \mathbf{U}_{x}\right]_{x} .
$$

It is shown (see Ladidus [14]) that this new difference scheme (obtained by adding the artificial viscosity) satisfies the same conservation law that the previous equation did. The values of the constant $\nu$ used varied from method to method. This is discussed in the section on numerical results. This artificial viscosity was not added in the smooth regions and not applied to the first component (mass) equation of (5). The artificial viscosity is not applied to the mass equation so as to minimize the amount of mass diffused across the contact discontinuity. 


\section{Harten's Corrective Method of Artificial Compression}

In this section we discuss the Artificial Compression Method (ACM) developed by Harten [9]. This method is designed to be used in conjunction with an already existing finite difference scheme. The purpose of this method is to sharpen the regions which contain discontinuities whether shocks or contact discontinuities.

Only the basic idea of the ACM will be discussed for the case of a single conservation law. Let $u(x, t)$ be a solution of the conservation law

$$
u_{t}+f(u)_{x}=0
$$

which contains a discontinuity $\left(u_{L}(t), u_{R}(t), S(t)\right)$, where $u_{L}$ and $u_{R}$ are the values on the left and right of the jump and $S$ is the speed of the discontinuity. The discontinuity is either a shock or a contact. Assume, without loss of generality that at any given time $t$ the solution $u$ does not take on any values between $u_{L}(t)$ and $u_{R}(t)$. Consider the function $g(u, t)$ with properties

$$
\begin{array}{ll}
g(u, t) \operatorname{sgn}\left[u_{R}(t)-u_{L}(t)\right]>0 & \text { for } u \in\left(u_{L}(t), u_{R}(t)\right), \\
g(u, t) \equiv 0 & \text { for } u \notin\left(u_{L}(t), u_{R}(t)\right) .
\end{array}
$$

This function $g$ will be called an artificial compression flux.

It can be seen that $u$ is also a solution of the conservation law

$$
u_{t}+(f(u) \cdot g(u, t))_{x}=0
$$

By (23) we see that when $u$ is smooth Eq. (24) is identical with Eq. (21) and the shock speed $S(t)$ remains the same. Finally it is observed (from (22)) that if $\left(u_{L}, u_{R}, S\right)$ is a shock or contact for Eq. (21) then it is a shock for the modified equation (24).

The artificial compression method solves the modified equation (24) rather than the original equation (21). For a complete discussion of the implementation of the method see Harten [9].

Let $\tilde{\mathbf{u}}_{i}^{n+1}$ represent the approximate solution vector to (5) obtained by using any one of the above first order difference methods. In solving the modified system (analogous to (24)) we use operator splitting. We first define the difference representation $\mathbf{g}_{i}$ of the artificial compression flux $g$,

$$
\mathbf{g}_{i}=\alpha_{i} \Delta_{i}
$$

where

$$
\Delta_{i}=\tilde{\mathbf{u}}_{i+1}-\tilde{\mathbf{u}}_{i-1}
$$

and

$$
\alpha_{i}=\max \left\{0, \min _{k}\left[\frac{\min \left(\left|\delta_{i+\frac{1}{k}}^{k}\right|, \delta_{i-1}^{k} \cdot \operatorname{sgn}\left(\delta_{i+\frac{1}{2}}^{k}\right)\right)}{\left|\delta_{i+\frac{1}{2}}^{k}\right|+\left|\delta_{i-\frac{1}{2}}^{k}\right|}\right]\right\}
$$


where $k$ refers to the $k$ th component of the $\tilde{\mathbf{u}}, \delta_{i+1}^{k}=\tilde{\mathbf{u}}_{i+1}^{(k)}-\tilde{\mathbf{u}}_{i}^{(k)}$. Let $\mathbf{S}_{i+\mathbf{t}}$ represent the vector whose $k$ th component is $\operatorname{sgn}\left(\delta_{i+\frac{1}{2}}^{k}\right)$. Then the difference scheme which applies the ACM to the given solution $\tilde{\mathbf{u}}_{i}^{n+1}$ is

$$
\begin{aligned}
\mathbf{u}_{i}^{n+1}= & \tilde{\mathbf{u}}^{n+1}-\frac{\Delta t}{2 \Delta x}\left(\mathbf{g}_{i+1}-\mathbf{g}_{i-1}\right) \\
& +\frac{\Delta t}{2 \Delta x}\left(\left|\mathbf{g}_{i+1}-\mathbf{g}_{i}\right| \mathbf{S}_{i+\frac{1}{2}}-\left|\mathbf{g}_{i}-\mathbf{g}_{i-\mathbf{1}}\right| \mathbf{S}_{i-\frac{1}{2}}\right) \\
= & \tilde{\mathbf{u}}_{i}^{n+1}-\frac{\Delta t}{2 \Delta x}\left(\mathbf{G}_{i+\frac{1}{2}}^{n}-\mathbf{G}_{i-\frac{1}{1}}^{n}\right),
\end{aligned}
$$

where $\mathbf{G}_{i+\frac{1}{2}}^{n}=\mathbf{g}_{i}{ }^{n}-\mathbf{g}_{i+1}^{n}-\left|\mathbf{g}_{i+1}^{n}-\mathbf{g}_{i}{ }^{n}\right| \mathbf{S}_{i+\frac{1}{\mathbf{1}}}$, applied componentwise. See Harten [8].

The method of artificial compression is designed for first-order schemes and cannot be applied directly to higher-order schemes. The idea of ACM is based on the existence of a viscous profile. See Harten [9]. Higher-order schemes introduce other flux terms so that one obtains different (nonphysical) speeds of propagation.

\section{Self-Adjusting Hybrid Schemes}

The idea of self-adjusting hybrid schemes was introduced by Harten and Zwas [12]. Consider a nonoscillatory first-order scheme $L_{1}$ and a $k$ th-order $(k \geqslant 2)$ scheme $L_{k}$,

$$
\begin{aligned}
& L_{1} u_{i}=u_{i}-(\Delta t / \Delta x)\left(f_{i+\underline{k}}^{1}-f_{i-\frac{1}{k}}^{1}\right), \\
& L_{k} u_{i}=u_{i}-(\Delta t / \Delta x)\left(f_{i+\underline{1}}^{k}-f_{i-\frac{1}{k}}^{k}\right) .
\end{aligned}
$$

So as not to violate the conservation, hybridize $L_{1}$ and $L_{k}$ through their numerical fluxes. Define the hybrid operator $L$ by

$$
L u_{i}=u_{i}-(\Delta t / \Delta x)\left(f_{i+\frac{1}{2}}-f_{i-\frac{1}{2}}\right)
$$

where

$$
f_{i+\frac{1}{1}}=\theta_{i+\frac{1}{2}} f_{i+\frac{1}{2}}^{1}+\left(1-\theta_{i+\frac{1}{2}}\right) f_{i+\frac{1}{2}}^{k},
$$

$\theta_{i+t}$ is a scalar quantity (called a switch) which satisfies $0 \leqslant \theta_{i+t} \leqslant 1$. At discontinuities the automatic switch is such that $\theta \approx 1$. Hence at the discontinuities the hybrid scheme is essentially the nonoscillatory first-order scheme.

Equation (30) can be written in the form

$$
L u_{i}=L_{k} u_{i}+\frac{\Delta t}{\Delta x}\left[\theta_{i+\frac{1}{3}}\left(f_{i+\frac{1}{2}}^{1}-f_{i+\frac{1}{2}}^{k}\right)-\theta_{i-\frac{1}{2}}\left(f_{i-\frac{1}{2}}^{1}-f_{i-\frac{1}{2}}^{k}\right)\right]
$$

so that if $\theta$ is $o\left(\Delta x^{p}\right)$ where the solution is smooth, then for $p \geqslant k-1$ we have

$$
L u_{i}=L_{k} u_{i}+o\left(\Delta x^{k+1}\right)
$$


There are many choices for such schemes. The scheme chosen here is discussed in Harten [10]. Taking $k=2$ we choose MacCormack's scheme and by adding the artificial viscosity term

$$
\frac{1}{8}\left(\theta_{i+1}\left(u_{i+1}-u_{i}\right)-\theta_{i-1}\left(u_{i}-u_{i-1}\right)\right)
$$

to MacCormack's scheme we obtain the first-order scheme.

The hybridized scheme becomes for system (5)

$$
\begin{aligned}
\mathbf{u}_{i}^{\overline{n+1}}= & \mathbf{u}_{i}{ }^{n} \quad \frac{\Delta t}{\Delta x^{-}\left(\mathbf{F}_{i+1}^{n}-\mathbf{F}_{i}{ }^{n}\right)} \\
\mathbf{u}_{i}^{n+1}= & \frac{1}{2}\left(\mathbf{u}_{i}^{\overline{n+1}}-\mathbf{u}_{i}{ }^{n}\right)-\frac{\Delta t}{2 \Delta x}\left(\overline{F_{i}^{n+1}}-\overline{F_{i-1}^{n+1}}\right) \\
& +\frac{1}{8}\left(\theta_{i+\frac{1}{2}}^{n}\left(\mathbf{u}_{i \nmid 1}^{n}-\mathbf{u}_{i}{ }^{n}\right)-\theta_{i-\frac{1}{2}}^{n}\left(\mathbf{u}_{i}{ }^{n}-\mathbf{u}_{i-1}^{n}\right)\right) .
\end{aligned}
$$

The stability condition for the first-order scheme is

$$
\max (|u|+c) \frac{\Delta t}{\Delta x} \leqslant \frac{3^{1 / 2}}{2},
$$

this being stricter than the stability condition for MacCormack's scheme. So this is the stability condition for the hybrid scheme.

It remains to describe how switch $\theta$ is chosen. There are many possible choices, the one selected is described in Harten [10]. Let $\Delta_{i+1}=p_{i+1}-\rho_{i}$. Define

$$
\begin{aligned}
& \hat{\theta}_{i}=\left|\frac{\left|\Delta_{i+\frac{1}{1}}\right|-\left|\Delta_{i-\frac{1}{1}}\right|}{\left|\Delta_{i+1}\right|+\left|\Delta_{i-\frac{1}{2}}\right|}\right|^{p}, \quad \text { for } \quad\left|\Delta_{i+1}\right|+\left|\Delta_{i-\frac{1}{2}}\right|>\epsilon, \\
& =0, \quad \text { otherwise. }
\end{aligned}
$$

In this case $p=1$ and $\epsilon>0$ is chosen as a measure of negligible variation in the density $\rho$. We define the switch $\theta$ by

$$
\theta_{i+\frac{1}{t}}=\max \left(\hat{\theta}_{i}, \hat{\theta}_{i+1}\right) .
$$

Since in areas which contain a discontinuity the hybrid scheme is about first order we may apply the artificial compression method discussed above. However, the ACM must not be used in smooth regions. For this purpose the switch is used again, i.e., Eq. (27b) may be replaced with

$$
\mathbf{u}_{i}^{n+1}=\tilde{\mathbf{u}}_{i}^{n+1}-\frac{\Delta t}{2 \Delta x}\left(\theta_{i+\frac{1}{2}}^{n} \mathbf{G}_{i+\frac{1}{2}}^{n}-\theta_{i-\frac{1}{2}}^{n} \mathbf{G}_{i-\frac{1}{2}}^{n}\right) .
$$




\section{Antidiffusion Method of Boris and Book}

In this section we shall discuss briefly the antidiffusion method developed by Boris and Book [1]. The purpose of this special technique known as "flux correction" is to achieve high resolution without oscillations.

It can be shown that a first-order difference scheme can be represented by an equation of the form

$$
u_{t}+f(u)_{x}=\Delta t\left[g(u, \Delta t / \Delta x) u_{x}\right]_{x}
$$

where $g(u, \Delta t / \Delta x)$ is the coefficient of the diffusion term.

The basis of the antidiffusion method is to use a stable modification of a diffusive difference scheme. Let the original scheme be represented by (39). The modification is represented by

$$
u_{t}+f(u)_{x}=\Delta t\left[(g(u, \Delta t / \Delta x)-r(u, \Delta t / \Delta x)) u_{x}\right]_{x}
$$

where $r$ is a positive function. One can introduce the antidiffusion term by operator splitting. The first step consists of solving

$$
u_{t}+f(u)_{x}=0
$$

with the original difference scheme, say $\tilde{\mathfrak{u}}_{i}^{n+1}=L u_{i}{ }^{n}$. Then in the second step let $A$ be a difference operator approximating the diffusion equation

$$
u_{t}+\Delta t\left[r(u, \Delta t / \Delta x) u_{x}\right]_{x}=0
$$

The second step is the antidiffusion step, which is unstable by itself since it approximates the backward heat equation. We define

$$
u_{i}^{n+1}=A \tilde{\mathbf{u}}_{i}^{n+1}=A L u_{i}^{n}
$$

It can be seen that if

$$
g(u, \Delta t / \Delta x)-r(u, \Delta t / \Delta x) \geqslant 0
$$

then the combined scheme $A L$ is stable. However, (43) places more of a restriction on $\Delta t / \Delta x$ than the stability condition for $L$.

We chose for $L$ the two-step Lax-Wenfroff scheme. Following Boris et al. [2], the procedure is

$$
\begin{aligned}
& u_{i+\frac{1}{2}}^{n+\frac{1}{2}}=\frac{1}{2}\left(\mathbf{u}_{i}^{n}+\mathbf{u}_{i+1}^{n}\right)-\frac{\Delta t}{2 \Delta x}\left(\mathbf{F}_{i+1}^{n}-\mathbf{F}_{i}^{n}\right), \\
& \tilde{\mathbf{u}}_{i}^{n+1}=\mathbf{u}_{i}^{n}-\frac{\Delta t}{\Delta x}\left(\mathbf{F}_{i+\frac{1}{2}}^{n+\frac{1}{2}}-\mathbf{F}_{i-\frac{1}{2}}^{n+\frac{1}{2}}\right)
\end{aligned}
$$




$$
\begin{aligned}
& \hat{\mathbf{u}}_{i}^{n+1}=\tilde{\mathbf{u}}_{i}^{n+1}+\eta\left(\mathbf{u}_{i+1}^{n}-2 \mathbf{u}_{i}^{n}+\mathbf{u}_{i-1}^{n}\right), \\
& \mathbf{u}_{i}^{n+1}=\hat{\mathbf{u}}_{i}^{n+1}-\left(f_{i+\frac{1}{2}}^{c}-f_{i-\frac{1}{2}}^{c}\right),
\end{aligned}
$$

where

$$
\begin{gathered}
\hat{\Delta}_{i+\frac{1}{2}}=\eta\left(\tilde{\mathbf{u}}_{i+1}^{n+1}-\tilde{\mathbf{u}}_{i}^{n+1}\right), \\
\Delta_{i \mid \frac{1}{1}}=\hat{\mathbf{u}}_{i \mid 1}^{n+1}-\hat{\mathbf{u}}_{i}^{n+1}, \\
f_{i+\frac{3}{2}}^{c}=\operatorname{sgn}\left(\hat{\Delta}_{i+\frac{1}{2}}\right) \max \left\{0, \min \left[\operatorname{sgn}\left(\hat{\Delta}_{i+\frac{1}{2}}\right) \Delta_{i-\frac{1}{2}},\left|\hat{\Delta}_{i+\frac{1}{2}}\right|, \operatorname{sgn}\left(\hat{\Delta}_{i+\frac{1}{2}}\right) \Delta_{i+\frac{1}{2}}\right]\right\} .
\end{gathered}
$$

The parameter $\eta$ is the diffusion/antidiffusion coefficient. The stability condition is

$$
\max (|u|+c)(\Delta t / \Delta x) \leqslant 1
$$

\section{Hyman's Predictor-Corrector Method}

In [13] Hyman describes a predictor-corrector type scheme. The spatial derivatives are approximated by a second-order difference operator while the time derivative (or time integrator) uses the improved Euler scheme. The improved Euler scheme combines a first-order explicit predictor with a second-order trapazoidal rule corrector.

For stability and to insure proper entropy production an artificial viscosity term is added. The artificial viscosity term used is similar to that used by Rusanov [21].

The scheme is given by

$$
\begin{aligned}
\mathbf{u}_{i}^{n+\frac{1}{2}} & =\mathbf{u}_{i}^{n}-\Delta t\left(D \mathbf{F}_{i}^{n}-\delta\left(\boldsymbol{\phi}_{i+\frac{1}{2}}^{n}-\boldsymbol{\phi}_{i-\frac{1}{2}}^{n}\right)\right) \\
& =\mathbf{u}_{i}{ }^{n}-\Delta t \mathbf{P}_{i}^{n}, \\
\mathbf{u}_{i}^{n+1} & =\mathbf{u}_{i}^{n}-(\Delta t / 2)\left(D \mathbf{F}_{i}^{n+\frac{1}{2}}+\mathbf{P}_{i}{ }^{n}\right),
\end{aligned}
$$

where

$$
\begin{aligned}
D \mathbf{F}_{i}{ }^{n} & =(1 / 12 \Delta x)\left(-\mathbf{F}_{i+2}^{n}+8 \mathbf{F}_{i+1}^{n}-8 \mathbf{F}_{i-1}^{n}+\mathbf{F}_{i-2}^{n}\right), \\
\boldsymbol{\phi}_{i+\frac{1}{2}}^{n} & =(1 / 4 \Delta x)\left(\alpha_{i+1}^{n}+\alpha_{i}^{n}\right)\left(\mathbf{u}_{i+1}^{n}-\mathbf{u}_{i}{ }^{n}\right), \\
\alpha_{i}{ }^{n} & =(u+c)_{i}^{n},
\end{aligned}
$$

and $c$ is the local sound speed.

The stability of the scheme depends on the number of applications of the corrector $(45 \mathrm{~b})$ and on $\delta$. We took as the stability condition

$$
\max (|u|+c)(\Delta t / \Delta x) \leqslant 1
$$

In order to maintain stability, the artificial viscosity must not be completely removed in the smooth regions. However, it can be reduced in these regions by using a type of 
switch. The one chosen was suggested by Hyman [13]. Replace $\phi_{i+\frac{1}{2}}^{n}$ in (45a) by $\beta \phi_{i+\frac{1}{2}}^{n}$ where

$$
\begin{aligned}
\beta & =\frac{1}{3}, & & \text { if } \quad \alpha_{i+1}^{n}>\alpha_{i}{ }^{n}+(\Delta x / 3) \\
& =1, & & \text { otherwise. }
\end{aligned}
$$

This type of switch greatly reduces the smearing of the contact discontinuity as well as the shock wave. This switch is a type of artificial compression.

\section{The Shock Tube Problem}

Figure 2 represents the initial conditions in a shock tube. A diaphragm at $x_{0}$ separates two regions (regions 1 and 5 ) which have different densities and pressures. The two regions are in a constant state. The initial conditions are $p_{1}>p_{5}, \rho_{1}>\rho_{5}$, and $u_{1}=u_{5}=0$; i.e., both fluids are initially at rest. At time $t>0$ (see Fig. 3 ) the diaphragm is broken. Consider the case before any wave has reached the left or right boundary. Points $x_{1}$ and $x_{2}$ represent the location of the head and tail of the rarefaction wave (moving to the left). Although the solution is continuous in this region

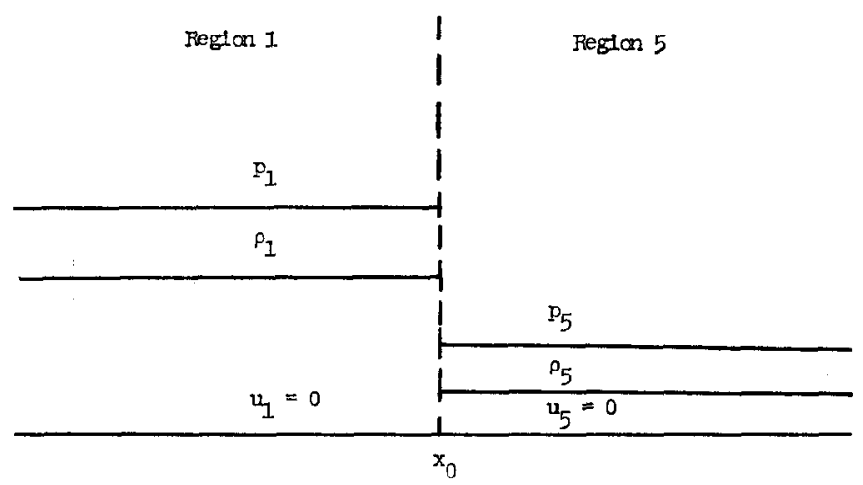

FIG. 2. Shock tube at $t=0$.

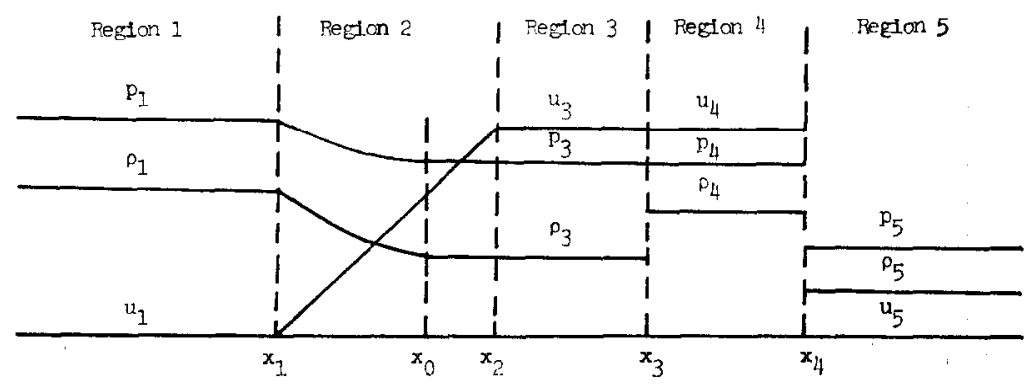

FIG. 3. Shock tube at $t>0$. 
(region 2) some of the derivatives of the fluid quantities may not be continuous The point $x_{3}$ is the position that an element of fluid initially at $x_{0}$ has reached by time $t$. Point $x_{3}$ is called a contact discontinuily. It is seen that across a contact discontinuity the pressure and the normal component of velocity are continuous. However, the density and the specific energy are not continuous across a contact discontinuity. Point $x_{4}$ is the location of the shock wave (moving to the right). Across a shock all of the quantities $(\rho, m, e$, and $p)$ will in general be discontinuous.

In the study of the above numerical methods the following test problem was considered: $\rho_{1}=1.0, p_{1}=1.0, u_{1}=0.0, \rho_{5}=0.125, p_{5}=0.1$, and $u_{5}=0$. The ratio of specific heats $\gamma$ was chosen to be 1.4. In all of the calculations $\Delta x=0.01$. For the Rusanov scheme the value of $\omega$ (see Table I) was taken to be 1.0. In the scheme of Boris and Book the parameter $\eta$ was taken to be 0.125 . For Hyman's scheme the value of $\delta$ was taken to be 0.8 . The constant in the artificial viscosity term $\nu$ was taken to be 1.0 in all but one case. Also the value of $\sigma$ (see Table I) was taken to be 0.9 .

In Glimm's original construction a new value of $\xi$ was chosen for each grid point $i$ and each time level $n$. The practical effect of such a choice with finite $\Delta x$ is disastrous since our initial data is not close to constant (which was an assumption made by Glimm). In fact, if $\xi$ is chosen for each $i$ and $n$, it is possible that a state will propagate to the left and to the right and thus create a spurious state. An improvement due to
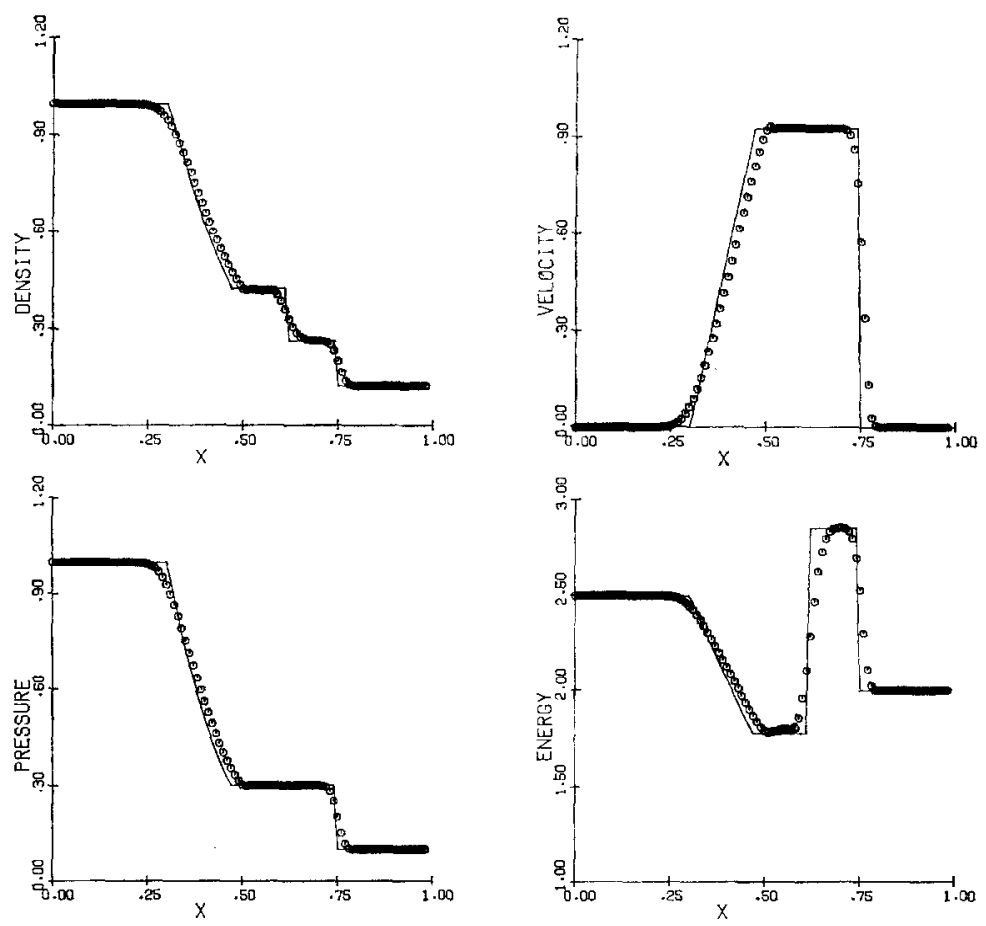

Fig. 4. Godunov's method. 

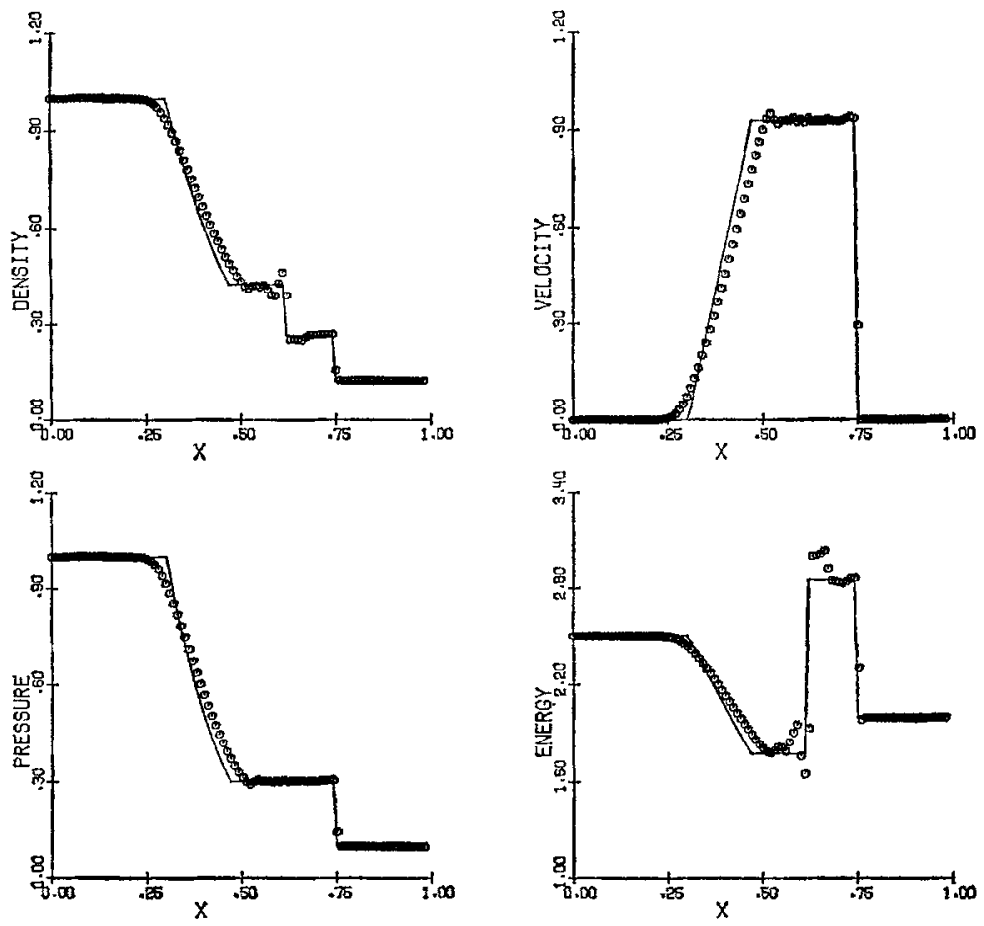

FIG. 5. Godunov's method with $A C M$.
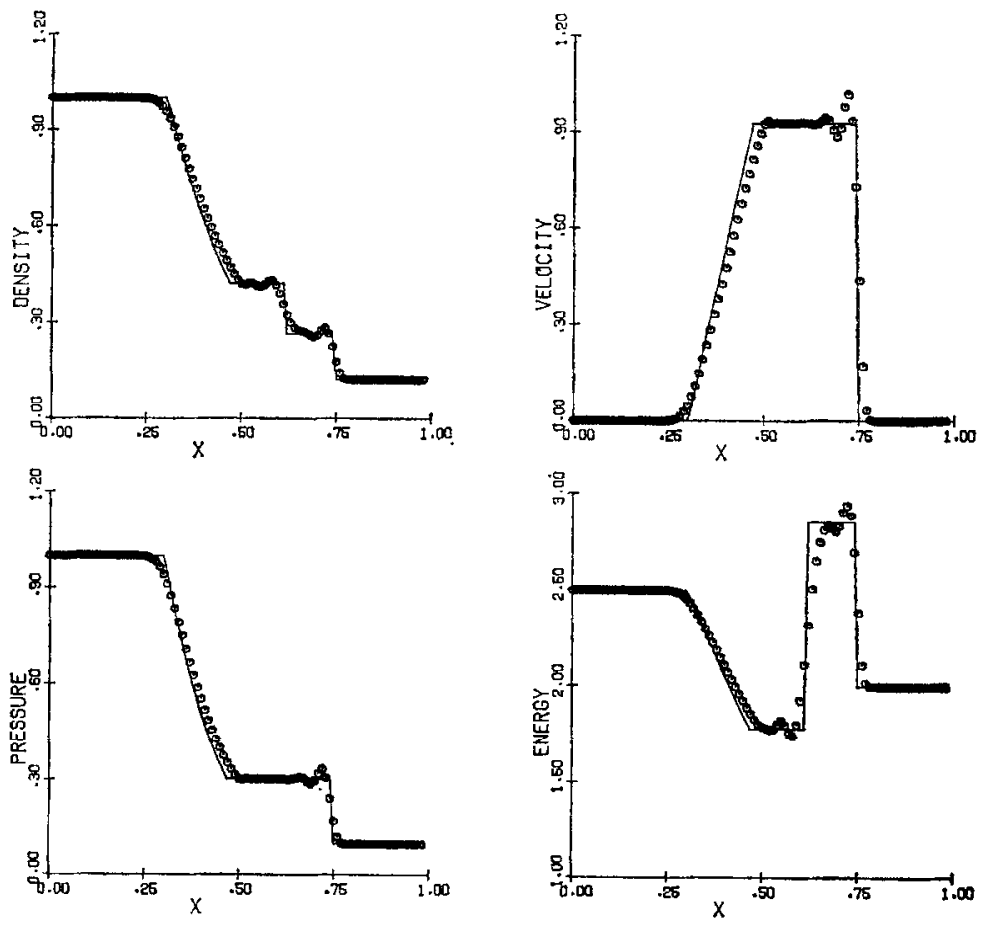

Fig. 6. Two-step Lax-Wendroff method. 
Chorin [3] is to choose $\xi_{n}$ only once per time step (hence the subscript $n$ ). The details of the method of selection of the random number are found in Chorin [3] and Sod [22].

Figure 4 indicates the results using the first-order accurate Godunov scheme. The corners at the endpoints of the rarefaction wave are rounded. The constant state between the contact discontinuity and the shock has not been fully realized. The transition of the contact discontinuity occupies seven to eight zones while the transition of the shock occupies five to six zones.

Figure 5 indicates the results using the Godunov scheme with artificial compression. It should be noted that for this case the constant in the artificial term was taken to be 2.0 to ensure that the solution before application of artificial compression was oscillation free. For the artificial compression cannot be applied in the presence of oscillations. The corners at the endpoints of the rarefaction wave are still rounded, since the artificial compression method is not applied in smooth regions. There is a slight undershoot at the right corner of the rarefaction. Also there are oscillations at the contact discontinuity. The transition of the contact discontinuity occupies three to four zones while the transition of the shock occupies only one to two zones.

Figure 6 shows the results of the two-step Lax-Wendroff scheme. There are very slight overshoots at the contact discontinuity and more noticeable overshoots at the shock. The rarefaction wave is quite accurate. The corners at the endpoints of the rarefaction are only slightly rounded. The transition of the contact discontinuity occupies six to eight zones while the shock wave occupies four to six zones. It is observed that the plots in Fig. 6 are quite similar to those in Fig. 7 obtained by MacCormack's method.

Figure 7 represents the results of the second-order MacCormack scheme. There are slight overshoots at the contact discontinuity and more noticeable overshoots at the shock wave. The rarefaction wave is quite accurate. The corners at the endpoints of the rarefaction are only slightly rounded. The transition of the contact discontinuity occupies seven to eight zones while the transition of the shock occupies five to six zones.

Figure 8 represents the first-order accurate Rusanov scheme. The contact discontinuity is barely visible in the density profile. The corners at the endpoints of the rarefaction wave are extremely rounded. The constant state between the contact discontinuity and the shock wave is barely existent. The transition of the contact discontinuity occupies 14-16 zones and the transition of the shock occupies 6-8 zones. This scheme is extremely diffusive.

Figure 9 represents the Rusanov scheme with artificial compression. The results with artificial compression are greatly improved. The corners at the endpoints of the rarefaction wave are still rounded since the artificial compression method is not applied in this area. The constant state between the contact discontinuity and the shock is much more visible. The transition of the contact discontinuity occupies two to three zones while that of the shock wave occupies only one to two zones.

Figure 10 represents the upwind difference scheme. It is observed that between the left constant state and the left endpoint of the rarefaction wave is a shock (discontinuity). This is clearly a nonphysical solution. This is a result of the method used 

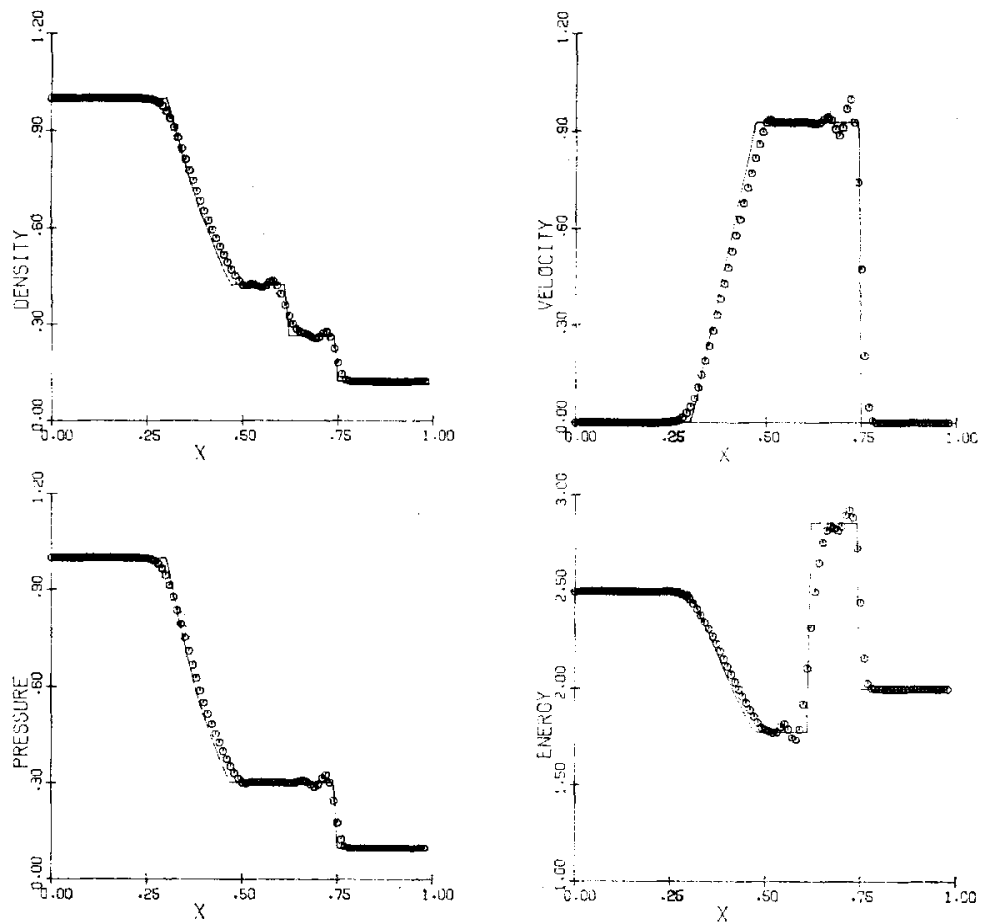

FIG. 7. MacCormack's method.
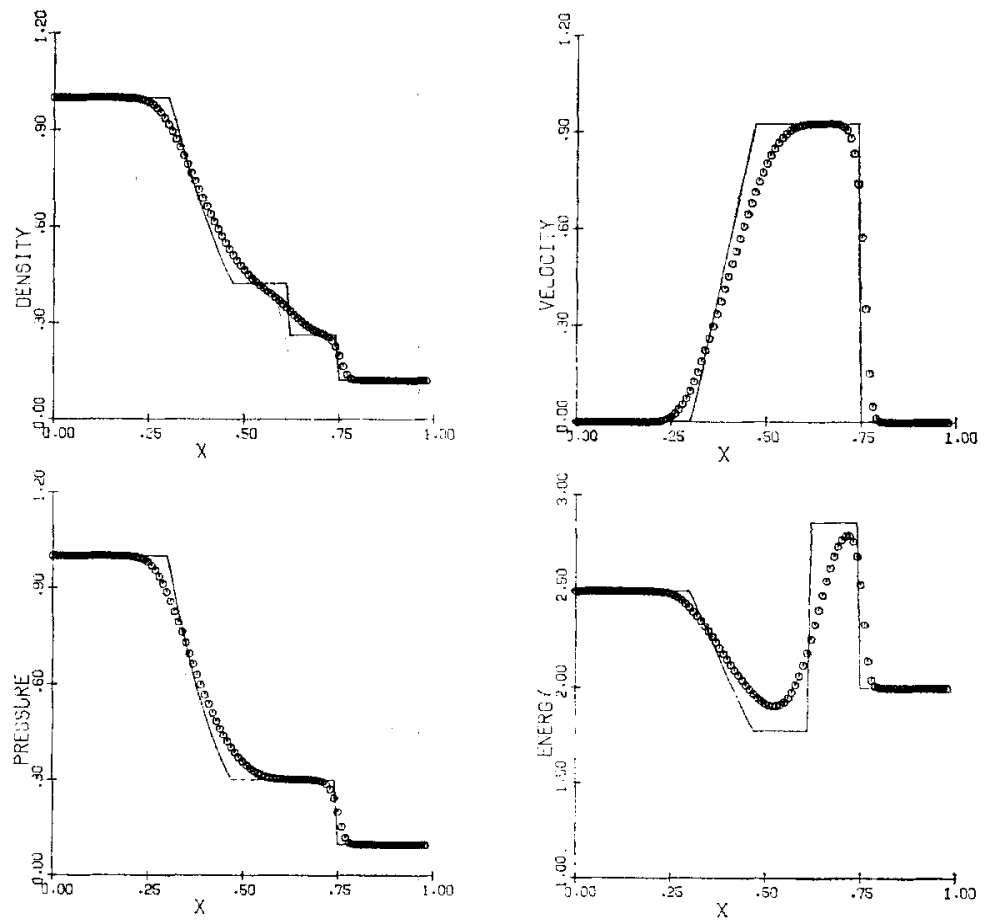

Fig. 8. Rusanov's first-order method. 

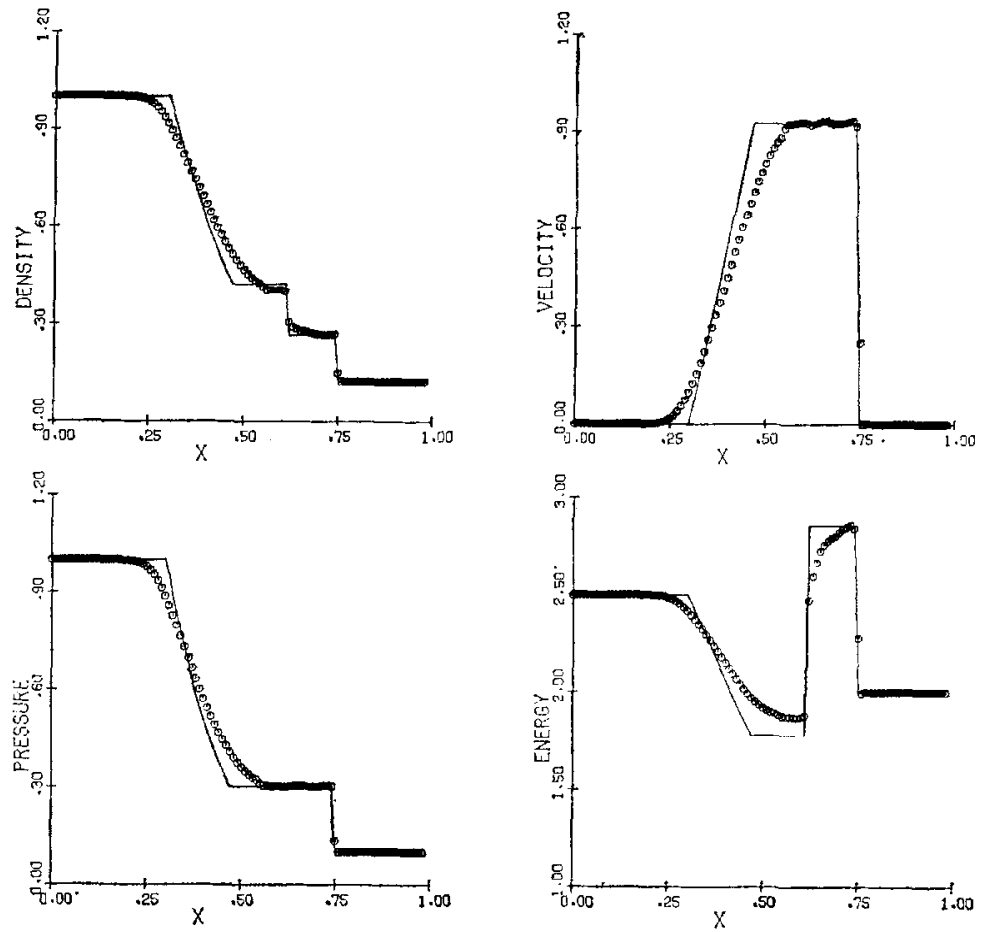

FIG. 9. Rusanov's first-order method with $A C M$.
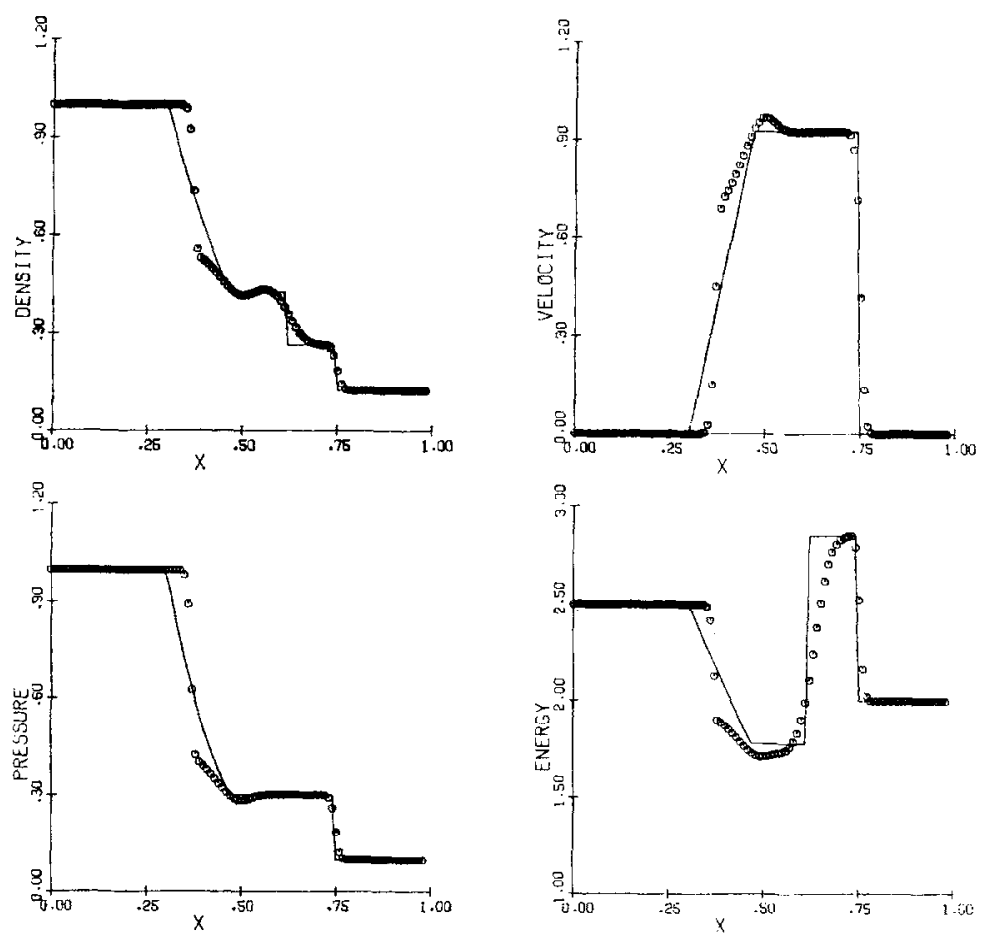

FIG. 10 Upwind difference method. 

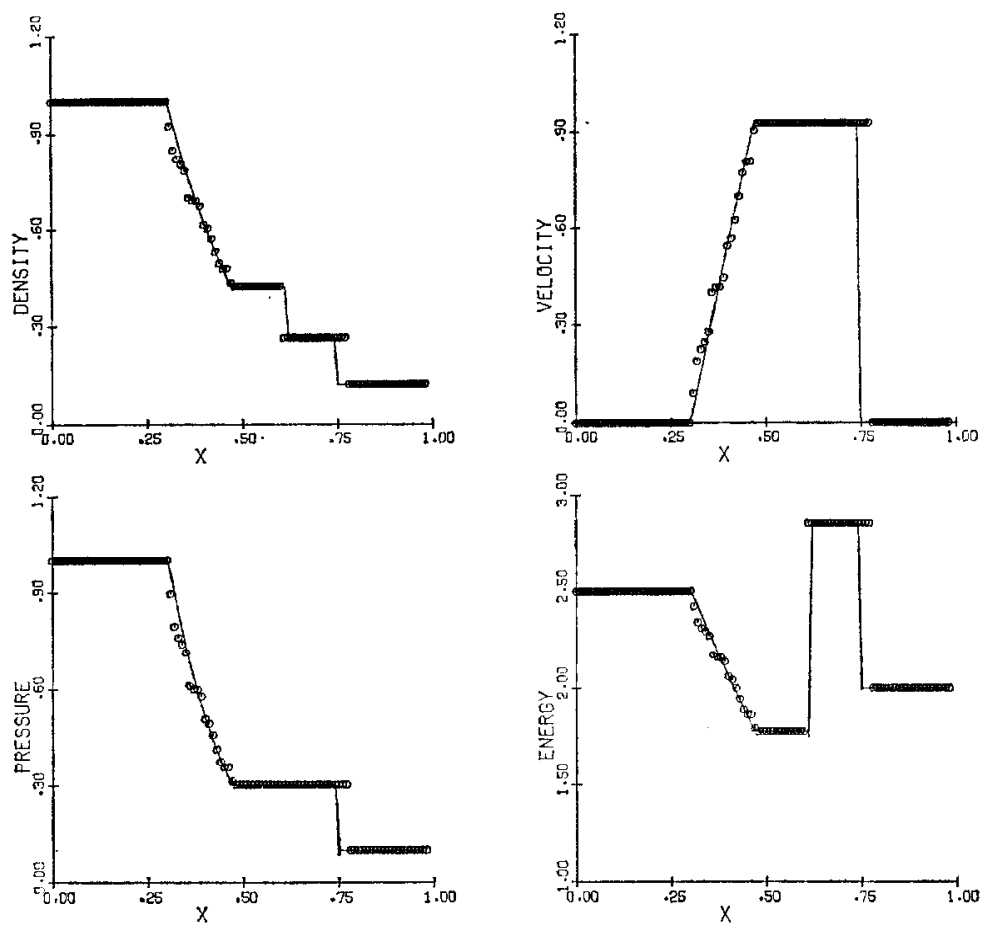

FIG. 11. Glimm's method.

to stabilize the scheme, by using centered differences for the pressure term in the momentum equation.

Figure 11 shows the results of the Glimm scheme. The shock wave and the contact discontinuity have been computed with infinite resolution, i.e., the number of zones over which the variation occurs is zero. Due to the randomness of the method the positions of the shock and the contact discontinuity are not exact. However, on the average their positions are exact. The corners at the endpoints of the rarefaction wave are perfectly sharp. It is observed that the rarefaction is not smooth (due to the randomness), yet it is extremely close to the exact solution. The constant states are perfectly realized.

The Glimm scheme requires between two and three times as much time (see below) as the other finite difference schemes tested. However, the Glimm scheme requires far less spatial grid points for the same resolution. This is displayed in Table II, where nine interior grid points are used. All details are visible.

The Glimm scheme on the average is conservative. One other check on the accuracy is to use the conservation laws (mass, momentum, and energy). For example, the total mass is evaluated by

$$
Q_{o}=\sum_{i} \rho(i \Delta x) \Delta x
$$


TABLE II

Profiles Obtained by Glimm's Method for Nine Interior Grid Points

\begin{tabular}{cccccc}
\hline$x$ & $\rho$ & $u$ & $p$ & $e$ & $\Gamma_{+}{ }^{a}$ \\
0.1 & 1.000 & 0.000 & 1.000 & 2.500 & 2.958 \\
0.2 & 1.000 & 0.000 & 1.000 & 2.500 & 2.958 \\
0.3 & 0.869 & 0.164 & 0.822 & 2.363 & 2.958 \\
0.4 & 0.426 & 0.927 & 0.303 & 1.778 & 2.958 \\
0.5 & 0.426 & 0.927 & 0.303 & 1.778 & 2.958 \\
0.6 & 0.426 & 0.927 & 0.303 & 1.778 & 2.958 \\
0.7 & 0.426 & 0.927 & 0.303 & 1.778 & 2.958 \\
0.8 & 0.266 & 0.927 & 0.303 & 2.853 & 3.624 \\
0.9 & 0.125 & 0.000 & 0.100 & 2.000 & 2.646 \\
\hline
\end{tabular}

${ }^{a} \Gamma_{+}$is the Riemann invariant $(c /(\gamma-1))+(u / 2)$, where $c$ is the local sound speed.

TABLE III

Total Mass, Momentum, and Energy for Glimm's Scheme

\begin{tabular}{rccc}
\hline$t / \Delta t$ & $Q_{\rho}$ & $Q_{m}$ & $Q_{e}$ \\
\hline 1 & 0.547 & 0.018 & 2.213 \\
2 & 0.550 & 0.019 & 2.217 \\
3 & 0.554 & 0.032 & 2.218 \\
4 & 0.550 & 0.039 & 2.219 \\
5 & 0.552 & 0.047 & 2.223 \\
6 & 0.550 & 0.059 & 2.222 \\
7 & 0.549 & 0.070 & 2.221 \\
8 & 0.550 & 0.079 & 2.224 \\
9 & 0.545 & 0.090 & 2.232 \\
10 & 0.546 & 0.097 & 2.247 \\
11 & 0.548 & 0.110 & 2.258 \\
12 & 0.545 & 0.119 & 2.267 \\
13 & 0.549 & 0.122 & 2.266 \\
14 & 0.552 & 0.136 & 2.266 \\
15 & 0.549 & 0.143 & 2.269 \\
16 & 0.553 & 0.149 & 2.275 \\
17 & 0.550 & 0.158 & 2.266 \\
18 & 0.546 & 0.164 & 2.267 \\
19 & 0.550 & 0.178 & 2.267 \\
20 & 0.543 & 0.190 & 2.272 \\
& & & \\
\hline
\end{tabular}



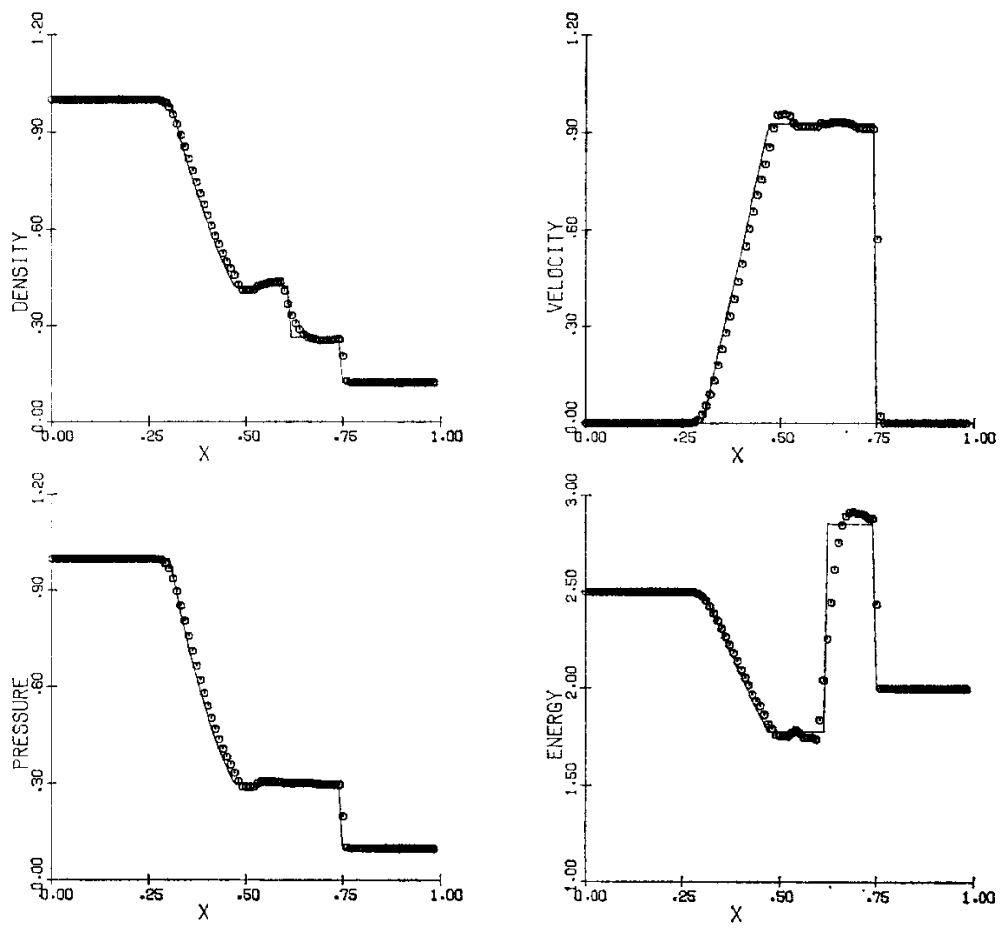

FIG. 12. Antidiffusion method.
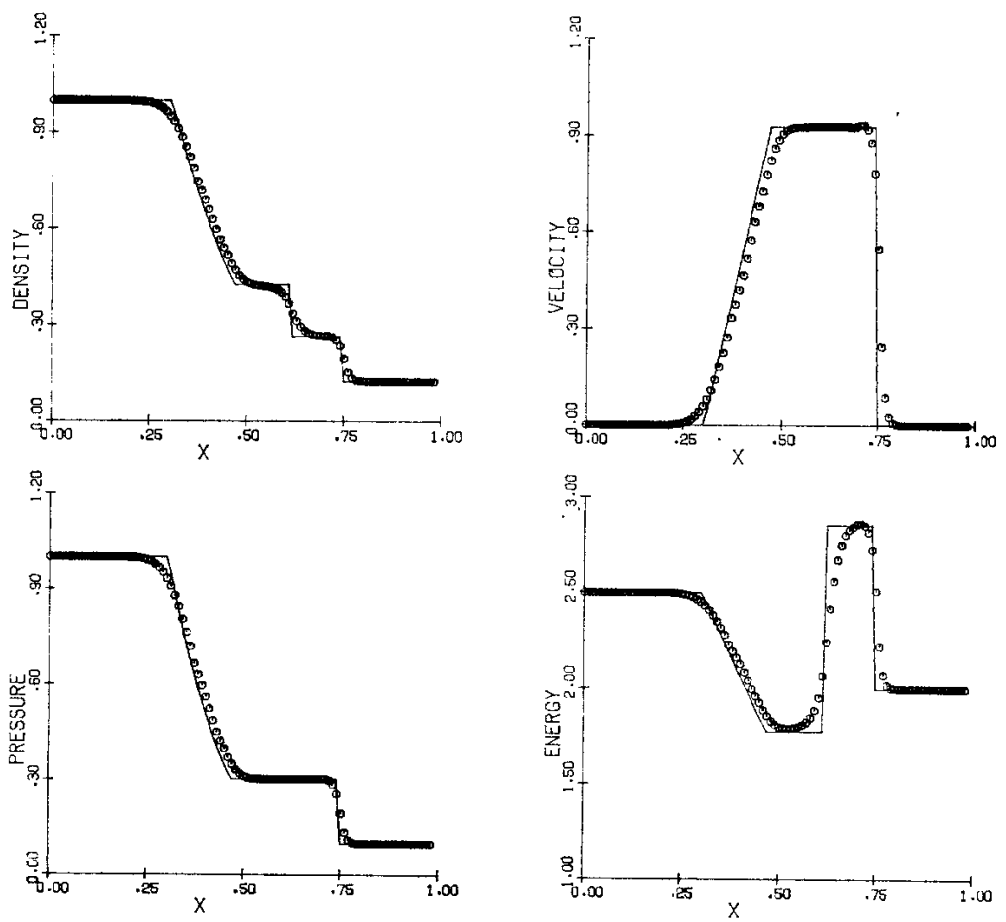

Fig. 13. Hybrid method. 

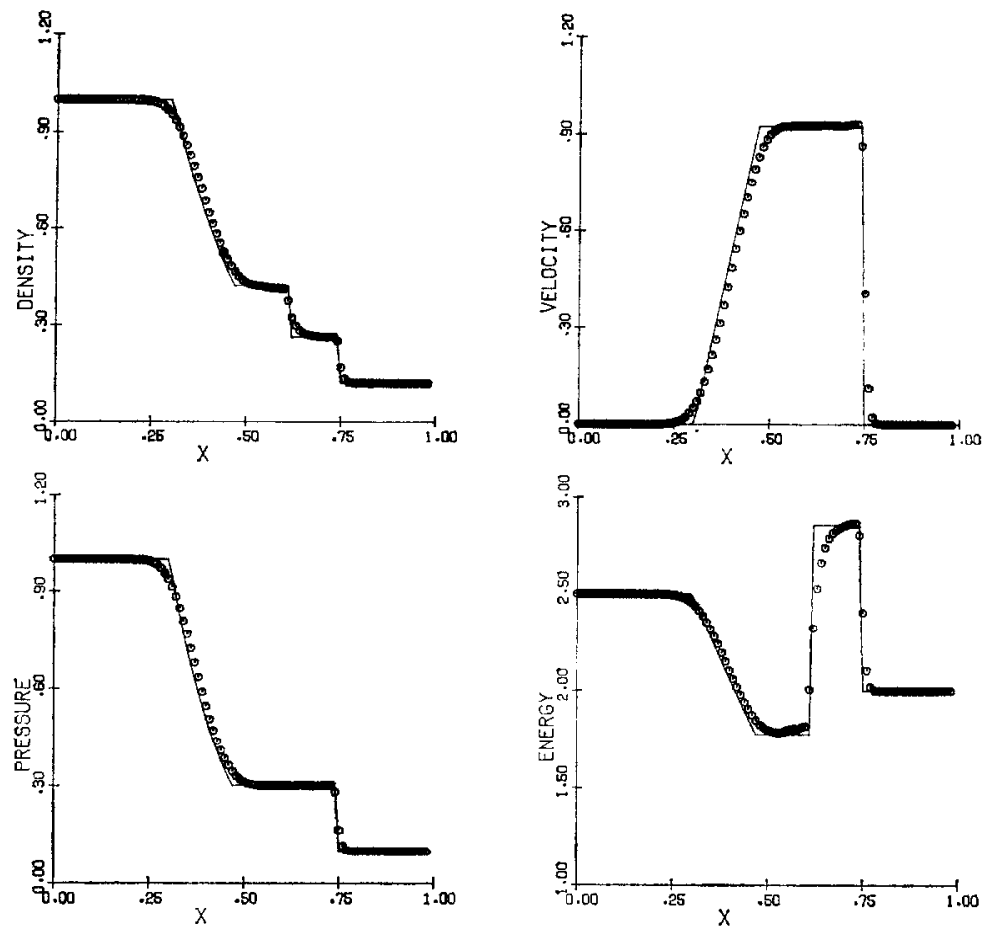

Fig. 14. Hybrid method with $A C M$.
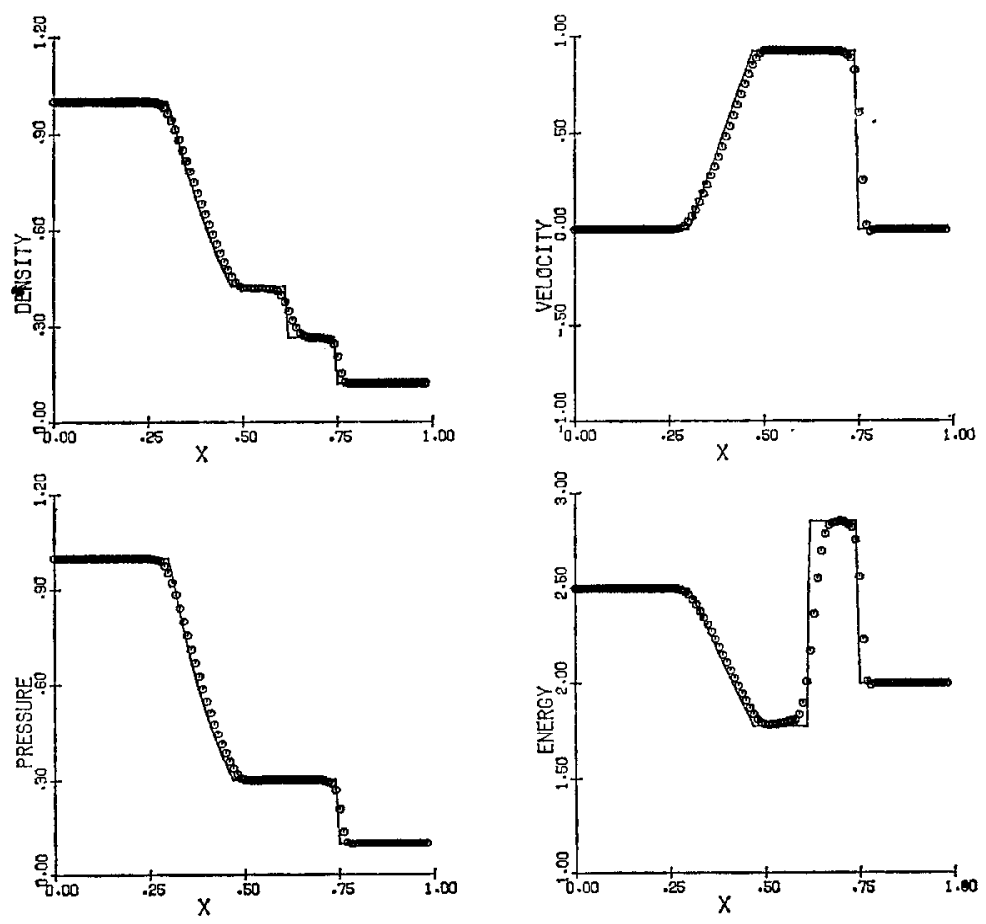

FIG. 15. Hyman's predictor-corrector method. 
In Table III the values of the total mass, momentum, and energy are displayed. The mass and the energy are seen to be conserved on the average, i.e., there are fluctuations but they are contained within a small interval. The momentum is seen to increase linearly on the average (allowing for fluctuations).

Figure 12 shows the results of the antidiffusion method of Boris and Book applied to the two-step Lax-Wendroff scheme. There is a slight overshoot at the right corner of the rarefaction. The rarefaction wave is very accurately computed. The corners at the endpoints of the rarefaction are only slightly rounded. The constant state between the contact discontinuity and the shock wave is only partially realized. The transition of the contact discontinuity occupies five to seven zones and the transition of the shock occupies one to two zones. The resolution is much better than the two-step Lax-Wendroff scheme alone (see Fig. 6).

Figure 13 represents the hybrid scheme (35) and (36) of Harten and Zwas. The solution is free of oscillations. The corners at the endpoints of the rarefaction wave are only slightly rounded. The constant state between the contact discontinuity and the shock is only partly realized. The transition of the contact discontinuity occupies eight to nine zones and the transition of the shock occupies five to six zones.

Figure 14 represents the hybrid scheme of Harten and Zwas with the use of artifical compression. Since the artifical compression is not applied in smooth regions the rarefaction is the same as in Fig. 13. The transition of the contact discontinuity occupies three to four zones and the transition of the shock wave occupies two to three zones.

Figure 15 represents the results of Hyman's predictor-corrector scheme, where the corrector has been applied once. The solution is oscillation free. The corners at the

TABLE IV

Running Time per Time Step (in sec) on CDC 6600

\begin{tabular}{lcc}
\hline Schemes & Without $A C M$ & With $A C M$ \\
\hline Godunov & 0.226 & 0.247 \\
Lax-Wendroff & 0.226 & - \\
MacCormack & 0.224 & - \\
Rusanov & 0.224 & 0.240 \\
Upwind & 0.225 & - \\
Glimm & 0.364 & - \\
Antidiffusion & 0.242 & - \\
Hybrid & 0.258 & 0.269 \\
Hyman & 0.276 & - \\
\hline
\end{tabular}

a Times include computation of exact solution, calls to printing, and plotting routines, which were the same for all cases. 
endpoints of the rarefaction are almost perfectly sharp. The constant states between the rarefaction and the contact discontinuity and between the contact discontinuity are extremely well defined. The transition of the contact discontinuity occupies six to eight zones while the transition of the shock occupies three to four zones.

The timing results for all of the methods are listed in Table IV. The times are for 100 spatial grid points. The only substantial difference in timing is between Glimm's scheme and the other finite difference schemes. For Glimm's scheme requires between two and three times as much time. However, Glimm's scheme can give the same resolution with far less points (as seen in Table II). From the point of view of the least number of grid points per desired resolution, the Glimm scheme can be seen to be much faster.

\section{Conclusions}

Of all the finite difference schemes tested, without the use of corrective procedures, Godunov's and Hyman's methods produced the best results.

It is obvious from the figures that the Glimm scheme gives the best resolution of the shocks and contact discontinuities. Glimm's scheme is at best first-order accurate (see Chorin [3]) so that boundary conditions are easily handled.

It is possible that the rarefaction wave obtained by Glimm's method can be smoothed out by a type of averaging. This is presently being considered.

The hybrid method of Harten and Zwas combines first- and high-order schemes in such a way as to extract the best features of both. The high-order scheme produces better approximations to the smooth parts of the flow.

The corrective procedures of Boris and Book and Harten improve the resolution of a given scheme. The artificial compression method being restricted to first-order schemes except when used in conjunction with the hybrid type schemes produces far better results than the antidiffusion method of Boris and Book. Both methods are easily added to existing programs (as a subroutine). The antidiffusion method requires slightly more storage than the artificial compression method since the former must retain two time levels of information for the computation of intermediate results (Eq. (44c)).

A major disadvantage of the antidiffusion method of Boris and Book, the hybrid scheme of Harten and Zwas, and the artificial compression method of Harten is that there are a number of parameters to be chosen, which depend on the given problem. In the antidiffusion method the coefficient of diffusion/antidiffusion must be chosen. The value of this parameter can greatly affect the results. In the hybrid scheme a tolerance must be chosen for the automatic switch which is taken to be a measure of negligible variation in entropy or density for example. This tolerance depends on the given problem. In the artificial compression method a test must be included to locate the rarefaction (and other smooth regions). Many of the standard tests fail to work well enough for the use of artificial compression.

; With the method described for solving the Riemann problem in the Glimm scheme, 
it can only be used for the equations of gas dynamics in rectangular coordinates. It is possible to generalize Glimm's method to other coordinate systems and different equations. See Harten and Sod [11].

The applicability of Glimm's method to other geometries has only just started to be explored. One successful application is to the equations of gas dynamics for a cylindrically or spherically symmetric flow. See Sod [23].

The usefulness of Glimm's method in the analysis of reacting gas flow is given in Chorin [4] and Sod [24]. In Chorin's paper examples are given of deflagration and detonation waves, with infinite and finite rates of reaction.

Since the Godunov iteration procedure provides an exact solution (up to the tolerence prescribed to terminate the iteration) to the Riemann problem, the onedimensional shock tube problem can be viewed as a "sitting duck" for Glimm's or any other numerical method using solutions of Riemann problems. However, it is observed that due to the randomness the results of Glimm's method are not exact.

The true value of a numerical method should be measured by its ability to solve multidimensional problems. For such problcms, Glimm's method or others which depend on the solution of one-dimensional Riemann problems may not have any advantage over the other methods considered here. This is presently being studied.

\section{APPENDIX: Implementation OF GLimm's Method}

In this appendix we discuss the equations required for the computer implementation of Glimm's method.

As in Fig. 1, the fluid initially at $x \leqslant 0$ is separated from the fluid initially at $x>0$ by a slip line $d x / d t=u_{*}$. There are a toal of 10 cases to consider.

I. The sample point $\xi_{n} \Delta x$ lies to the left of the slip line $\left(\xi_{n} \Delta x<u_{*} \Delta t / 2\right)$.

(a) If the left wave is a shock wave $\left(p_{*}>p_{l}\right)$ and (1) if $\xi_{n} \Delta x$ lies to the left of the shock line $d x / d t=U_{l}$, we have $\rho=\rho_{l}, u=u_{l}$, and $p=p_{l}$, (2) if $\xi_{n} \Delta x$ lies to the right of the shock line $d x / d t=U_{l}$, we have $\rho=\rho_{*}, u=u_{*}, p=p_{*}$, where $\rho_{*}$ can be obtained from (13)

$$
\rho_{*}=M_{l} /\left(U_{l}-u_{*}\right)
$$

(b) If the left wave is a rarefaction wave $\left(p_{*} \leqslant p_{l}\right)$. Define the sound speed to be $c=(\gamma p / \rho)^{\frac{1}{2}}$. The rarefaction wave is bounded on the left by the line defined by $d x / d t=u_{l}-c_{l}$, where $c_{l}=\left(\gamma p_{l} / \rho_{l}\right)^{\frac{1}{2}}$, and on the right by the line defined by $d x / d t=$ $u_{*}-c_{*}$, where $c_{*}=\left(\gamma p_{*} / \rho_{*}\right)^{\frac{1}{2}}$. The flow is adiabatic in smooth regions, so in this region $A(S)$ in (4) is a constant, denoted by $A$, and we obtain the isentropic law $p=A p^{v} \cdot \rho_{*}$ is obtained by using the isentropic law

$$
p_{l} \rho_{l}^{-\gamma}=p_{*} \rho_{*}^{-\gamma}=A .
$$


Then we obtain from (46)

$$
\rho_{*}=\left(p_{*} / A\right)^{\mathrm{I} / \nu} .
$$

(1) If $\xi_{n} \Delta x$ lies to the left of the rarefaction wave, then $\rho=\rho_{l}, u=u_{l}$, and $p=p_{l}$.

(2) If $\xi_{n} \Delta x$ lies inside the left rarefaction wave, we equate the slope of the characteristic $d x / d t=u-c$ to the slope of the line through the origin and $\left(\xi_{n} \Delta x\right.$, $\Delta t / 2)$, obtaining

$$
u-c=2 \xi_{n} \Delta x / \Delta t
$$

With the constancy of the Riemann invariant

$$
2 c(\gamma-1)^{-1}+u=2 c_{l}(\gamma-1)^{-1}+u_{l},
$$

the isentropic law, and the definition of $c$, we can obtain $\rho, u$, and $p$. Using the isentropic law we obtain

$$
p=p_{\imath} \rho_{l}^{-\gamma} \rho^{\gamma}=A \rho^{\gamma} .
$$

Using Eq. (50) we obtain, by solving for $c$,

$$
c=c_{l}+\frac{\gamma-1}{2}\left(u_{l}-u\right) .
$$

By substitution of (52) into (49) and solving for $u$ we obtain

$$
u=\frac{2}{\gamma-1}\left(\frac{2 \xi_{n} \Delta x}{\Delta t}+c_{l}+\frac{(\gamma-1)}{2} u_{l}\right) .
$$

By substitution of (53) into (52) $c$ is obtained; by substitution of (52) into the definition of $c$ and solving for $\rho$ we obtain

$$
\rho=\left(c^{2} / \gamma A\right)^{1 / \gamma-1} .
$$

(3) If $\xi_{n} \Delta x$ lies to the right of the left rarefaction wave we obtain $\rho=\rho_{*}$, $u=u_{*}$, and $p=p_{*}$.

II. The sample point $\xi_{n} \Delta x$ lies to the right of the slip line $\left(\xi_{n} \Delta x \geqslant u_{*} \Delta t / 2\right)$.

(a) If the right wave is a shock wave $\left(p_{*}>p_{r}\right)$ and (1) if $\xi_{n} \Delta x$ lies to the left of the shock line defined by $d x / d t=U_{r}$, we have $\rho=\rho_{*}, u=u_{*}$, and $p=p_{*}$, where $\rho_{*}$ is obtained from (15)

$$
\rho_{*}=-M_{r} /\left(u_{*}-U_{r}\right)
$$


(2) If $\xi_{n} \Delta x$ lies to the right of the shock line defined by $d x / d t=U_{r}$, we have $\rho=\rho_{r}, u=u_{r}$, and $p=p_{r}$.

(b) If the right wave is a rarefaction wave $\left(p_{*} \leqslant p_{r}\right)$. The rarefaction wave is bounded on the left by the line defined by $d x / d t=u_{*}+c_{*}$, where $c_{*}=\left(\gamma p_{*} / \rho_{*}\right)^{\frac{1}{2}}$ and $\rho_{*}$ can be obtained from the isentropic law

$$
p_{r} \rho_{r}^{-\gamma}=p_{*} \rho_{*}^{-\gamma}=A .
$$

Then we obtain from (55)

$$
\rho_{*}=\left(p_{*} / A\right)^{1 / \nu}
$$

and on the right by the line defined by $d x / d t=u_{r} \mid c_{r}, c_{r}=\left(\gamma p_{r} / \rho_{r}\right)^{\frac{1}{2}}$.

(1) If $\xi_{n} \Delta x$ lies to the left of the rarefaction wave, then $\rho=\rho_{*}, u=u_{*}$, and $p=p_{*}$.

(2) If $\xi_{n} \Delta x$ lies inside the right rarefaction wave, we equate the slope of the characteristic $d x / d t=u+c$ to the slope of the line through the origin and $\left(\xi_{n} \Delta x\right.$, $\Delta t / 2)$, obtaining

$$
u+c=2 \xi_{n} \Delta x / \Delta t
$$

With the constancy of the Riemann invariant

$$
2 c(\gamma-1)^{-1}-u=2 c_{r}(\gamma-1)^{-1}-u_{r}
$$

the isentropic law, and the definition of $c$, we can obtain $\rho, u$, and $p$. Using the isentropic law we obtain

$$
p=p_{r} \rho_{r}^{-\gamma} \rho^{\nu}=A \rho^{\nu} .
$$

Using Eq. (58) we obtain, by solving for $c$

$$
c=c_{r}+\frac{\gamma-1}{2}\left(u-u_{r}\right)
$$

Substitution of (60) into (57) and solving for $u$ we obtain

$$
u=\frac{2}{\gamma+1}\left(\frac{2 \xi_{n} \Delta x}{\Delta t}-c_{r}+\frac{\gamma-1}{2} u_{r}\right)
$$

By substitution of (61) into (60) $c$ is obtained; by substitution of (59) into the definition of $c$ and solving for $\rho$ we obtain

$$
\rho=\left(c^{2} / \gamma A\right)^{1 / \gamma-1} .
$$



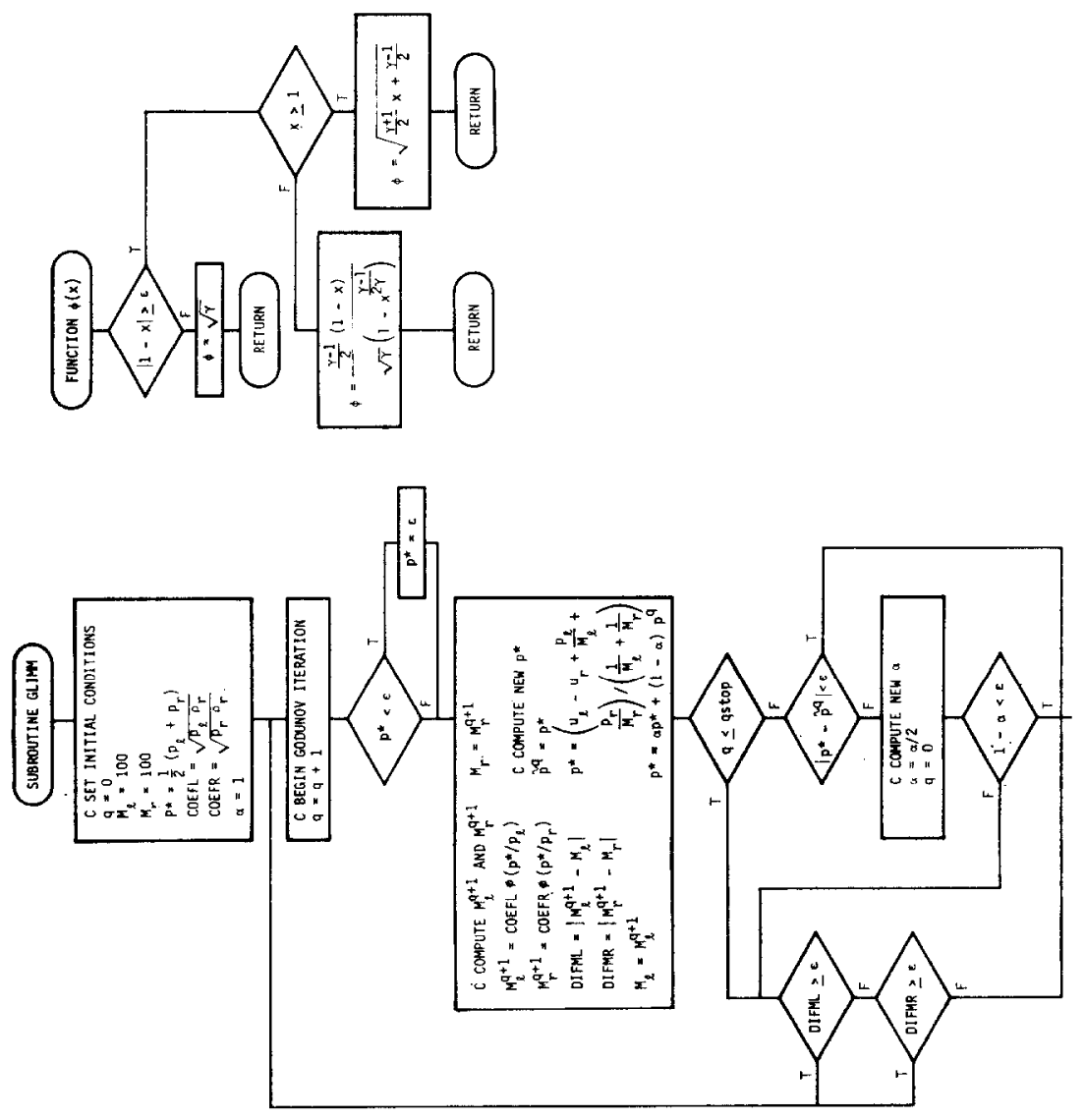


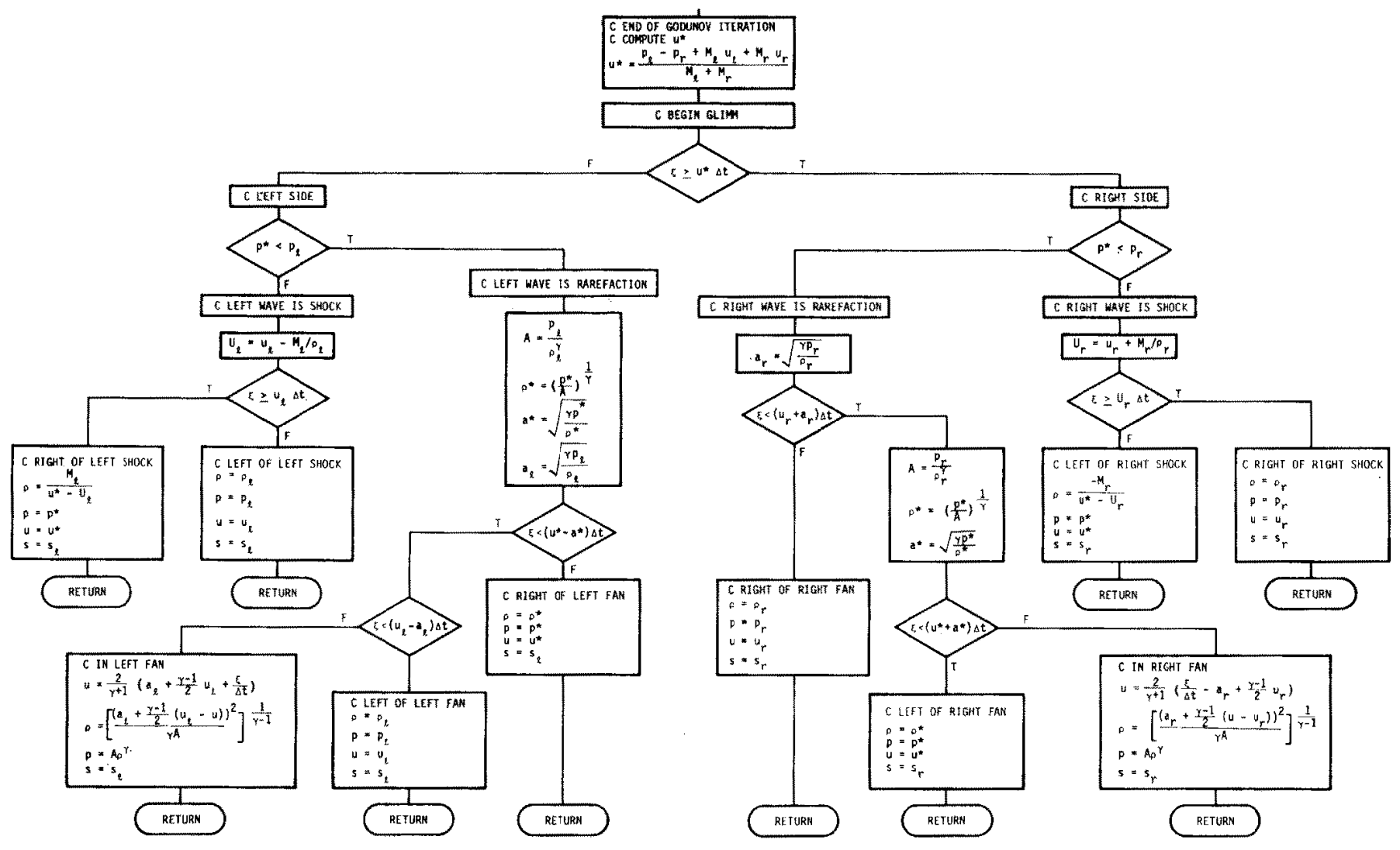

Fig. 16. Flow chart of Glimm's method. 
(3) If $\xi_{n} \Delta x$ lies to the right of the right rarefaction wave we obtain $\rho=\rho_{r}$, $u=u_{r}$, and $p=p_{r}$.

Equations (46)-(62) are the key to the programming of Glimm's method. For a summary see the flow chart (Fig. 16).

\section{ACKNOWLEDGMENTS}

The author would like to thank Professor Alexandre Chorin, Mr. Phillip Colella, Dr. Amiram Harten, and Dr. James M. Hyman for a number of helpful discussions and comments. The author would also like to thank one of the editors, Dr. Raymond Chin, for his assistance. This work was supported in part by the National Science Foundation, Grant MCS76-07039, the Energy Research and Development Administration under Contract No. EY-76-C-02-3077 at the Courant Institute, and the Energy Research and Development Administration under Contract No. W-7405-Eng-48 at Lawrence Livermore Laboratory.

\section{REFERENCES}

1. J. P. Boris ANd D. L. Book, J. Computational Phys. 11 (1973), 38.

2. J. P. Boris, D. L. Book, AND K. J. HaIN, J. Computational Phys. 18 (1975), 248.

3. A. J. Chorin, J. Computational Phys. 22 (1976), 517.

4. A. J. Chorin, J. Computational Phys. 25 (1977), 253.

5. S. K. Godunov, Mat. Sbornik 47 (1959), 271.

6. S. K. Godunov, A. V. Zabrodin, AND G. P. Prokopov, J. Comp. Math. Math. Phys. USSR 1 (1962), 1187.

7. J. Glimm, Comm. Pure Appl. Math. 18 (1965), 697.

8. A. HARTEN, "The Method of Artificial Compression," AEC Research and Development Report C00-3077-50, New York University, 1974.

9. A. Harten, Comm. Pure Appl. Math. 30 (1977), 611.

10. A. Harten, The artificial compression method for shocks and contact discontinuities. III. Self-adjusting hybrid schemes, to appear.

11. A. HARTEN AND G. SoD, A generalized version of Glimm's method, to appear.

12. A. Harten and G. Zwas, J. Computational Phys. 6 (1972), 568.

13. J. M. Hyman, On robust and accurate methods for the calculation of compressible fluid flows, I, to appear.

14. A. LAPIDUs, J. Computational Phys. 2 (1967), 154.

15. P. D. LaX, Comm. Pure Appl. Math. 7 (1954), 159.

16. P. D. LAX, Comm. Pure Appl. Math. 10 (1957), 537.

17. B. vaN LeER, J. Computational Phys, 3 (1969), 473.

18. R. MACCORMACK, "Proceedings of the Second International Conference on Numerical Methods in Fluid Dynamics," Lecture Notes in Physics, (M. Holt, Ed.), Vol. 8, Springer-Verlag, New York, 1971.

19. R. Richtmyer, "A Survey of Difference Methods for Non-Steady Fluid Dynamics," NCAR Technical Note 63-2, National Center for Atmospheric Research, Boulder, Colo., 1962.

20. R. Richtmyer and K. Morton, "Difference Methods for Initial-Value Problems," 2nd ed., Interscience, New York, 1967.

21. V. V. Rusanov, J. Comp. Math. Math. Phys. USSR, No. 2 (1962). 
22. G. A. SoD, "The Computer Implementation of Glimm's Method," UCID-17252, Lawrence Livermore Laboratory, University of California, 1976.

23. G. A. SoD, J. Fluid Mech. 83 (1977), 785.

24. G. A. SoD, A numerical model of unsteady combustion phenomena, to appear.

25. G. A. SoD, "Numerical Analysis with Application to Fluid Dynamics," Courant Institute Lecture Notes, to appear.

26. J. von NeumanN and R. D. Richtmyer, J. Appl. Phys. 21 (1950), 232.

27. S. E. BuCKLEY AND M. C. LeVeretT, Trans. AIME 146 (1942), 107. 